\title{
Iridium-Catalyzed Direct ortho-C-H Amidation of Benzaldehydes through N-Sulfonyl Imines as Mask
}

\author{
Yudong Li, Yadong Feng, Linhua Xu, Lianhui Wang, Xiuling Cui* \\ Engineering Research Center of Molecular Medicine of Ministry of Education, Key \\ Laboratory of Fujian Molecular Medicine, Key Laboratory of Xiamen Marine and Gene \\ Drugs, School of Biomedical Sciences, Huaqiao University, Xiamen 361021, P. R. China
}

Corresponding Author: Xiuling Cui,

Email: cuixl@hqu.edu.cn

Tel \& Fax: +86-592-6162996

Context

1. General information ----------------------------------------------------------------------S2

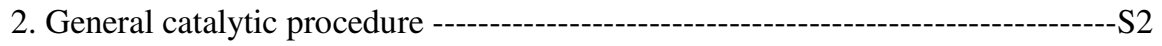

3. Characterization data for products --------------------------------------------------S2

4. Experiments with isotopically-labeled substrates -----------------------------------S6

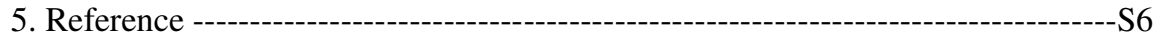

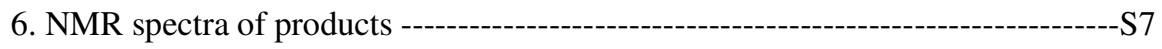




\section{General information}

All manipulations were conducted with a standard Schlenk technique under air atmosphere. Unless otherwise stated, all commercial materials and solvents were used directly without further purification. Commercially available chemicals were obtained from Energy Chemical, TCI, Alfa Aesar, J\&K. 1H and 13C NMR spectra were measured on a $400 \mathrm{MHz}$ Bruker spectrometer $\left({ }^{1} \mathrm{H}\right.$ $400 \mathrm{MHz},{ }^{13} \mathrm{C} 100 \mathrm{MHz}$ ), using $\mathrm{CDCl}_{3}$ as the solvent with tetramethylsilane (TMS) as the internal standard at room temperature. Chemical shifts are reported in ppm from tetramethylsilane with the

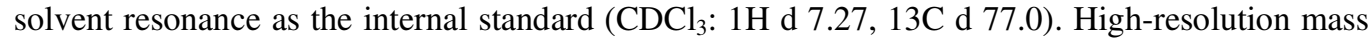
spectra (HRMS) were recorded using Agilent 6450 spectrometer. Column chromatography was performed on silica gel (70-230 mesh ASTM) using the reported eluents. Thin-layer chromatography (TLC) was carried out on $4 \times 15 \mathrm{~cm}$ plates with a layer thickness of $0.2 \mathrm{~mm}$ (silica gel $60 \mathrm{~F}_{254}$ ). Starting materials $\mathbf{1}^{1}$ and $\mathbf{2}^{2}$ were prepared according to the literatures.

\section{General catalytic procedure}

Under air atmosphere, a reaction tube $(25 \mathrm{~mL})$ equipped with a magnetic stirrer bar was charged with $\mathrm{N}$-Sulfonyl imine $1(0.2 \mathrm{mmol})$, sulfonyl azide $2(0.24 \mathrm{mmol}),\left[\mathrm{IrCp}^{*} \mathrm{Cl}_{2}\right]_{2}(1.6 \mathrm{mg}, 0.02$ mmol, $1 \mathrm{~mol} \%), \operatorname{AgNTf}_{2}(12.4 \mathrm{mg}, 0.32 \mathrm{mmol}, 8 \mathrm{~mol} \%)$, and 1,2-dichloroethane (1.0 mL). The reaction mixture was stirred at $60{ }^{\circ} \mathrm{C}$ for $12 \mathrm{~h}$. After cooled to room temperature, $2 \mathrm{M} \mathrm{HCl}(1 \mathrm{~mL})$ was added. The resulting mixture was stirred at room temperature for another $3 \mathrm{~h}$ and then extracted with ethyl acetate $(3 \times 10 \mathrm{~mL})$. The solvents were removed under reduced pressure, and the residue was purified by chromatography on silica gel (eluent: petroleum ether/ethyl acetate = 10:1) to give the desired product 3 .

\section{Characterization of the Products}

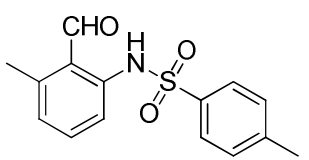

N-(2-formyl-3-methylphenyl)-4-methylbenzenesulfonamide (3aa) ${ }^{1}$

Yellow solid, $51.4 \mathrm{mg}, 89 \%$ yield, mp: $94-95{ }^{\circ} \mathrm{C} .{ }^{1} \mathrm{H}$ NMR $\left(400 \mathrm{MHz}, \mathrm{CDCl}_{3}\right) \delta 11.44(\mathrm{~s}, 1 \mathrm{H}), 10.35(\mathrm{~s}$, $1 \mathrm{H}), 7.78(\mathrm{~d}, J=8.0 \mathrm{~Hz}, 2 \mathrm{H}), 7.53(\mathrm{~d}, J=8.2 \mathrm{~Hz}, 1 \mathrm{H}), 7.37(\mathrm{t}, J=7.8 \mathrm{~Hz}, 1 \mathrm{H}), 7.26(\mathrm{~d}, J=7.8 \mathrm{~Hz}$, $2 \mathrm{H}), 6.87(\mathrm{~d}, J=7.1 \mathrm{~Hz}, 1 \mathrm{H}), 2.61(\mathrm{~s}, 3 \mathrm{H}), 2.38(\mathrm{~s}, 3 \mathrm{H}) ;{ }^{13} \mathrm{C} \mathrm{NMR}\left(101 \mathrm{MHz}, \mathrm{CDCl}_{3}\right) \delta 194.0,144.1$, $143.7,140.8,136.5,136.1,129.8,127.3,125.7,119.3,116.1,21.6,19.0$.<smiles>COc1ccc(S(=O)(=O)Nc2cccc(C)c2C=O)cc1</smiles>

N-(2-formyl-3-methylphenyl)-4-methoxybenzenesulfonamide (3ab) ${ }^{2}$

White solid, $54.3 \mathrm{mg}, 89 \%$ yield, mp: $145-148{ }^{\circ} \mathrm{C} .{ }^{1} \mathrm{H}$ NMR $\left(400 \mathrm{MHz}, \mathrm{CDCl}_{3}\right) \delta 11.40(\mathrm{~s}, 1 \mathrm{H})$, $10.35(\mathrm{~s}, 1 \mathrm{H}), 7.82(\mathrm{~d}, J=8.9 \mathrm{~Hz}, 2 \mathrm{H}), 7.52(\mathrm{~d}, J=8.4 \mathrm{~Hz}, 1 \mathrm{H}), 7.37(\mathrm{t}, J=8.0 \mathrm{~Hz}, 1 \mathrm{H}), 6.91(\mathrm{~d}, J=$ $8.9 \mathrm{~Hz}, 2 \mathrm{H}), 6.87(\mathrm{~d}, J=7.4 \mathrm{~Hz}, 1 \mathrm{H}), 3.83(\mathrm{~s}, 3 \mathrm{H}), 2.61(\mathrm{~s}, 3 \mathrm{H}) ;{ }^{13} \mathrm{C} \mathrm{NMR}\left(101 \mathrm{MHz}, \mathrm{CDCl}_{3}\right) \delta 194.0$, $163.2,143.7,140.9,136.1,131.0,129.5,125.7,119.3,116.1,114.3,55.6,19.0$.

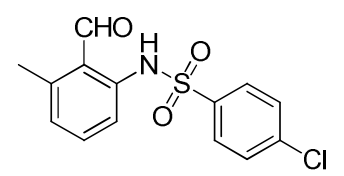

4-chloro-N-(2-formyl-3-methylphenyl)benzenesulfonamide (3ac)

White solid, $55.6 \mathrm{mg}, 90 \%$ yield, mp: $149-150{ }^{\circ} \mathrm{C} .{ }^{1} \mathrm{H}$ NMR $(400 \mathrm{MHz}, \mathrm{CDCl} 3) \delta 11.47$ (s, 1H), 10.35 (s, 1H), $7.82(\mathrm{~d}, \mathrm{~J}=8.6 \mathrm{~Hz}, 2 \mathrm{H}), 7.54(\mathrm{~d}, \mathrm{~J}=8.4 \mathrm{~Hz}, 1 \mathrm{H}), 7.43(\mathrm{~d}, \mathrm{~J}=8.7 \mathrm{~Hz}, 2 \mathrm{H}), 7.39$ (d, J = 8.2 Hz, 
$1 \mathrm{H}), 6.92(\mathrm{~d}, \mathrm{~J}=7.5 \mathrm{~Hz}, 1 \mathrm{H}), 2.63(\mathrm{~s}, 3 \mathrm{H}) ;{ }^{13} \mathrm{C} \mathrm{NMR}\left(101 \mathrm{MHz}, \mathrm{CDCl}_{3}\right) \delta 194.1,143.9,140.4,139.7$, 137.9, 136.2, 129.5, 128.7, 126.2, 119.5, 116.3, 19.0. HRMS (ESI) m/z calcd for $\mathrm{C}_{14} \mathrm{H}_{12} \mathrm{ClNO}_{3} \mathrm{~S}$ $(\mathrm{MNa}+)$ 332.0119, found 332.0124.

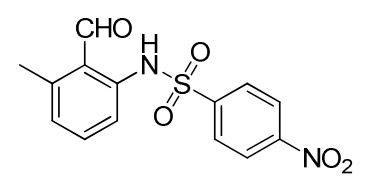

N-(2-formyl-3-methylphenyl)-4-nitrobenzenesulfonamide (3ad)

Yellow solid, $46.1 \mathrm{mg}, 72 \%$ yield, mp: $159-160{ }^{\circ} \mathrm{C} .{ }^{1} \mathrm{H}$ NMR $(400 \mathrm{MHz}, \mathrm{CDCl} 3) \delta 11.58(\mathrm{~s}, 1 \mathrm{H})$, $10.35(\mathrm{~s}, 1 \mathrm{H}), 8.30(\mathrm{~d}, \mathrm{~J}=8.7 \mathrm{~Hz}, 2 \mathrm{H}), 8.07(\mathrm{~d}, \mathrm{~J}=8.8 \mathrm{~Hz}, 2 \mathrm{H}), 7.57$ (d, J = 8.3 Hz, 1H), 7.43 (t, J = $8.0 \mathrm{~Hz}, 1 \mathrm{H}), 6.96(\mathrm{~d}, \mathrm{~J}=7.5 \mathrm{~Hz}, 1 \mathrm{H}), 2.63$ (s, 3H); 13C NMR (101 MHz, CDCl3) $\delta 194.3,150.3,145.1$, 144.2, 139.8, 136.4, 128.6, 126.9, 124.4, 119.7, 116.4, 19.0. HRMS (ESI) m/z calcd for $\mathrm{C}_{14} \mathrm{H}_{12} \mathrm{~N}_{2} \mathrm{O}_{5} \mathrm{~S}$ $(\mathrm{MNa}+)$ 343.0359, found 343.0361.<smiles>Cc1cccc(NS(=O)(=O)c2ccc(F)cc2)c1C=O</smiles>

4-fluoro-N-(2-formyl-3-methylphenyl)benzenesulfonamide (3ae)

Yellow solid, $47 \mathrm{mg}, 80 \%$ yield, mp: 97-98 ${ }^{\circ} \mathrm{C}$. ${ }^{1} \mathrm{H}$ NMR (400 MHz, CDCl3) $\delta 11.45$ (s, 1H), 10.35 (s, $1 \mathrm{H}), 7.94-7.87(\mathrm{~m}, 2 \mathrm{H}), 7.54(\mathrm{~d}, \mathrm{~J}=8.4 \mathrm{~Hz}, 1 \mathrm{H}), 7.40(\mathrm{t}, \mathrm{J}=8.0 \mathrm{~Hz}, 1 \mathrm{H}), 7.17-7.09(\mathrm{~m}, 2 \mathrm{H}), 6.91(\mathrm{~d}$, $\mathrm{J}=7.5 \mathrm{~Hz}, 1 \mathrm{H}), 2.63(\mathrm{~s}, 3 \mathrm{H}) ;{ }^{13} \mathrm{C} \mathrm{NMR}(101 \mathrm{MHz}, \mathrm{CDCl} 3) \delta 194.1,165.3(\mathrm{~d}, \mathrm{~J}=255.6 \mathrm{~Hz}), 143.9$, 140.5, 136.2, 135.5 (d, J = 3.3 Hz), $130.1(\mathrm{~d}, \mathrm{~J}=9.5 \mathrm{~Hz}), 126.2,119.5,116.5,116.3(\mathrm{~d}, \mathrm{~J}=6.0 \mathrm{~Hz})$, 19.0; ${ }^{19} \mathrm{~F}$ NMR $\left(376 \mathrm{MHz}, \mathrm{CDCl}_{3}\right) \delta-104.3$. HRMS (ESI) $\mathrm{m} / \mathrm{z}$ calcd for $\mathrm{C}_{14} \mathrm{H}_{12} \mathrm{FNO}_{3} \mathrm{~S}(\mathrm{MNa}+)$ 316.0414 , found 316.0420 .<smiles>Cc1cccc(NS(=O)(=O)c2ccccc2)c1C=O</smiles>

N-(2-formyl-3-methylphenyl)benzenesulfonamide (3af)

White solid, $52 \mathrm{mg}, 95 \%$ yield, mp: $122-123{ }^{\circ} \mathrm{C} .{ }^{1} \mathrm{H}$ NMR $\left(400 \mathrm{MHz}, \mathrm{CDCl}_{3}\right) \delta 11.47(\mathrm{~s}, 1 \mathrm{H}), 10.35(\mathrm{~s}$, $1 \mathrm{H}), 7.90(\mathrm{~d}, J=7.5 \mathrm{~Hz}, 2 \mathrm{H}), 7.55(\mathrm{t}, J=7.5 \mathrm{~Hz}, 2 \mathrm{H}), 7.47(\mathrm{t}, J=7.6 \mathrm{~Hz}, 2 \mathrm{H}), 7.38(\mathrm{t}, J=8.0 \mathrm{~Hz}, 1 \mathrm{H})$, $6.88(\mathrm{~d}, J=7.5 \mathrm{~Hz}, 1 \mathrm{H}), 2.61(\mathrm{~s}, 3 \mathrm{H}) ;{ }^{13} \mathrm{C} \mathrm{NMR}\left(101 \mathrm{MHz}, \mathrm{CDCl}_{3}\right) \delta 194.0,143.8,140.7,139.4,136.1$, 133.2, 129.1, 127.3, 125.9, 119.4, 116.2, 19.0. HRMS (ESI) m/z calcd for $\mathrm{C}_{14} \mathrm{H}_{13} \mathrm{NO}_{3} \mathrm{~S}(\mathrm{MH}+)$ 276.0689 , found 276.0694 .

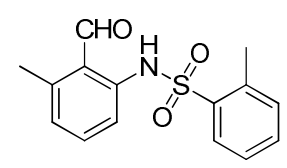

N-(2-formyl-3-methylphenyl)-2-methylbenzenesulfonamide (3ag)

White solid, $41.6 \mathrm{mg}, 72 \%$ yield, mp: $96-97{ }^{\circ} \mathrm{C} .{ }^{1} \mathrm{H}$ NMR $\left(400 \mathrm{MHz}, \mathrm{CDCl}_{3}\right) \delta 11.68(\mathrm{~s}, 1 \mathrm{H}), 10.41(\mathrm{~s}$, $1 \mathrm{H}), 8.12(\mathrm{~d}, J=7.8 \mathrm{~Hz}, 1 \mathrm{H}), 7.46(\mathrm{t}, J=7.3 \mathrm{~Hz}, 1 \mathrm{H}), 7.39(\mathrm{~d}, J=8.3 \mathrm{~Hz}, 1 \mathrm{H}), 7.33(\mathrm{dd}, J=17.4,9.8$ $\mathrm{Hz}, 3 \mathrm{H}), 6.85(\mathrm{~d}, J=7.1 \mathrm{~Hz}, 1 \mathrm{H}), 2.66(\mathrm{~s}, 3 \mathrm{H}), 2.64(\mathrm{~s}, 3 \mathrm{H}) ;{ }^{13} \mathrm{C} \mathrm{NMR}\left(101 \mathrm{MHz}, \mathrm{CDCl}_{3}\right) \delta 194.1$, 143.8, 140.8, 137.6, 137.4, 136.2, 133.3, 132.8, 130.1, 126.1, 125.3, 118.7, 115.0, 20.0, 19.1. HRMS (ESI) $\mathrm{m} / \mathrm{z}$ calcd for $\mathrm{C}_{15} \mathrm{H}_{15} \mathrm{NO}_{3} \mathrm{~S}(\mathrm{MH}+)$ 290.0845, found 290.0851 .<smiles>Cc1cccc(NS(=O)(=O)c2cccc(Cl)c2)c1C=O</smiles>

2-chloro-N-(2-formyl-3-methylphenyl)benzenesulfonamide (3ah)

White solid, $46.4 \mathrm{mg}, 75 \%$ yield, mp: $113-114{ }^{\circ} \mathrm{C} .{ }^{1} \mathrm{H}$ NMR $\left(400 \mathrm{MHz}, \mathrm{CDCl}_{3}\right) \delta 11.53(\mathrm{~s}, 1 \mathrm{H})$, $10.37(\mathrm{~s}, 1 \mathrm{H}), 7.89(\mathrm{~s}, 1 \mathrm{H}), 7.79(\mathrm{~d}, J=7.8 \mathrm{~Hz}, 1 \mathrm{H}), 7.53(\mathrm{dd}, J=7.3,3.5 \mathrm{~Hz}, 2 \mathrm{H}), 7.44-7.39(\mathrm{~m}, 2 \mathrm{H})$, $6.93(\mathrm{~d}, J=7.5 \mathrm{~Hz}, 1 \mathrm{H}), 2.64(\mathrm{~s}, 3 \mathrm{H}) ;{ }^{13} \mathrm{C} \mathrm{NMR}\left(101 \mathrm{MHz}, \mathrm{CDCl}_{3}\right) \delta 194.1,143.9,141.2,140.3,136.3$, 135.3, 133.3, 130.4, 127.3, 126.2, 125.4, 119.5, 116.2, 19.0. HRMS (ESI) m/z calcd for $\mathrm{C}_{14} \mathrm{H}_{12} \mathrm{ClNO}_{3} \mathrm{~S}$ $(\mathrm{MNa}+)$ 332.0119, found 332.0124. 
<smiles>Cc1cccc(NS(C)(=O)=O)c1C=O</smiles>

N-(2-formyl-3-methylphenyl)methanesulfonamide (3ai) ${ }^{3}$

Yellow solid, $40.5 \mathrm{mg}, 95 \%$ yield, mp: $94-95{ }^{\circ} \mathrm{C} .{ }^{1} \mathrm{H}$ NMR $\left(400 \mathrm{MHz}, \mathrm{CDCl}_{3}\right) \delta 11.23(\mathrm{~s}, 1 \mathrm{H}), 10.45$ $(\mathrm{s}, 1 \mathrm{H}), 7.60(\mathrm{~d}, J=8.4 \mathrm{~Hz}, 1 \mathrm{H}), 7.50(\mathrm{t}, J=8.0 \mathrm{~Hz}, 1 \mathrm{H}), 6.98(\mathrm{~d}, J=7.5 \mathrm{~Hz}, 1 \mathrm{H}), 3.11(\mathrm{~s}, 3 \mathrm{H}), 2.70(\mathrm{~s}$, $3 \mathrm{H}) ;{ }^{13} \mathrm{C}$ NMR (101 MHz, $\left.\mathrm{CDCl}_{3}\right) \delta$ 194.0, 144.2, 141.1, 136.5, 125.8, 119.1, 115.3, 40.3, 19.1.<smiles>Cc1ccc(S(=O)(=O)Nc2cccc(F)c2C=O)cc1</smiles>

N-(3-fluoro-2-formylphenyl)-4-methylbenzenesulfonamide (3ba)

Yellow solid, $53 \mathrm{mg}, 91 \%$ yield, mp: $132-133^{\circ} \mathrm{C} .{ }^{1} \mathrm{H}$ NMR $\left(400 \mathrm{MHz}, \mathrm{CDCl}_{3}\right) \delta 11.11(\mathrm{~s}, 1 \mathrm{H}), 10.29$ (s, 1H), $7.80(\mathrm{~d}, J=8.3 \mathrm{~Hz}, 2 \mathrm{H}), 7.54-7.43(\mathrm{~m}, 2 \mathrm{H}), 7.29(\mathrm{~d}, J=4.7 \mathrm{~Hz}, 2 \mathrm{H}), 6.82-6.75(\mathrm{~m}, 1 \mathrm{H})$, 2.40 (s, 3H); 13C NMR (101 MHz, CDCl3) $\delta 190.3$ (d, J = 12.2 Hz), 167.3, 164.7, 144.5, 141.4, 137.8 $(\mathrm{d}, \mathrm{J}=11.6 \mathrm{~Hz}), 136.1,129.9,127.3,113.2(\mathrm{~d}, \mathrm{~J}=3.7 \mathrm{~Hz}), 109.4(\mathrm{~d}, \mathrm{~J}=20.9 \mathrm{~Hz}), 21.6 ;{ }^{19} \mathrm{~F}$ NMR $(376$ $\left.\mathrm{MHz}, \mathrm{CDCl}_{3}\right) \delta$-120.3. HRMS (ESI) $\mathrm{m} / \mathrm{z}$ calcd for $\mathrm{C}_{14} \mathrm{H}_{12} \mathrm{FNO}_{3} \mathrm{~S}(\mathrm{MNa}+)$ 316.0414, found 316.0420.

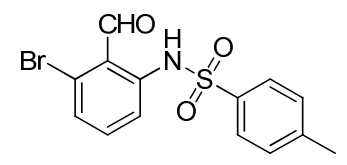

N-(3-bromo-2-formylphenyl)-4-methylbenzenesulfonamide (3ca)

Yellow solid, $62 \mathrm{mg}, 88 \%$ yield, mp: $145-146{ }^{\circ} \mathrm{C} .{ }^{1} \mathrm{H}$ NMR $(400 \mathrm{MHz}, \mathrm{CDCl} 3) \delta 11.39(\mathrm{~s}, 1 \mathrm{H})$, $10.37(\mathrm{~s}, 1 \mathrm{H}), 7.78(\mathrm{~d}, \mathrm{~J}=8.2 \mathrm{~Hz}, 2 \mathrm{H}), 7.67(\mathrm{dd}, \mathrm{J}=6.4,2.8 \mathrm{~Hz}, 1 \mathrm{H}), 7.32(\mathrm{~d}, \mathrm{~J}=3.6 \mathrm{~Hz}, 1 \mathrm{H}), 7.30(\mathrm{~d}$, $\mathrm{J}=5.8 \mathrm{~Hz}, 2 \mathrm{H}), 7.27(\mathrm{~s}, 1 \mathrm{H}), 2.40(\mathrm{~s}, 3 \mathrm{H}) ;{ }^{13} \mathrm{C} \mathrm{NMR}\left(101 \mathrm{MHz}, \mathrm{CDCl}_{3}\right) \delta 196.3,144.5,142.3,136.5$, 136.1, 130.1, 129.9, 128.1, 127.3, 118.2, 117.4, 21.6. HRMS (ESI) $\mathrm{m} / \mathrm{z}$ calcd for $\mathrm{C}_{14} \mathrm{H}_{12} \mathrm{BrNO}_{3} \mathrm{~S}$ $(\mathrm{MNa}+)$ 375.9613, found 375.9619.

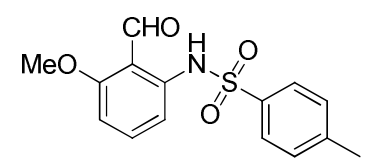

N-(2-formyl-3-methoxyphenyl)-4-methylbenzenesulfonamide (3da)

White solid, $50 \mathrm{mg}, 82 \%$ yield, mp: $144-146{ }^{\circ} \mathrm{C} .{ }^{1} \mathrm{H}$ NMR $(400 \mathrm{MHz}, \mathrm{CDCl} 3) \delta 11.54(\mathrm{~s}, 1 \mathrm{H}), 10.39$ $(\mathrm{s}, 1 \mathrm{H}), 7.78(\mathrm{~d}, \mathrm{~J}=8.1 \mathrm{~Hz}, 2 \mathrm{H}), 7.41(\mathrm{t}, \mathrm{J}=8.4 \mathrm{~Hz}, 1 \mathrm{H}), 7.28(\mathrm{~d}, \mathrm{~J}=7.5 \mathrm{~Hz}, 2 \mathrm{H}), 7.21(\mathrm{~d}, \mathrm{~J}=8.4 \mathrm{~Hz}$, $1 \mathrm{H}), 6.56(\mathrm{~d}, \mathrm{~J}=8.4 \mathrm{~Hz}, 1 \mathrm{H}), 3.87(\mathrm{~s}, 3 \mathrm{H}), 2.38(\mathrm{~s}, 3 \mathrm{H}) ;{ }^{13} \mathrm{C} \mathrm{NMR}\left(101 \mathrm{MHz}, \mathrm{CDCl}_{3}\right) \delta$ 193.0, 163.4, 144.1, 141.6, 137.6, 136.5, 129.7, 127.3, 110.9, 109.5, 104.9, 56.0, 21.6. HRMS (ESI) m/z calcd for $\mathrm{C}_{15} \mathrm{H}_{15} \mathrm{NO}_{4} \mathrm{~S}(\mathrm{MH}+)$ 306.0795, found 306.0800 .

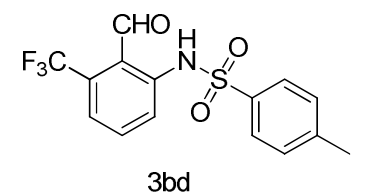

N-(2-formyl-3-(trifluoromethyl)phenyl)-4-methylbenzenesulfonamide (3ea)

Yellow solid, $54.8 \mathrm{mg}, 80 \%$ yield, mp: $115-117{ }^{\circ} \mathrm{C} .{ }^{1} \mathrm{H}$ NMR $\left(400 \mathrm{MHz}, \mathrm{CDCl}_{3}\right) \delta 11.51 \quad(\mathrm{~s}, 1 \mathrm{H})$, $10.28(\mathrm{~d}, J=1.5 \mathrm{~Hz}, 1 \mathrm{H}), 7.96(\mathrm{~d}, J=8.6 \mathrm{~Hz}, 1 \mathrm{H}), 7.80(\mathrm{~d}, J=8.2 \mathrm{~Hz}, 2 \mathrm{H}), 7.61(\mathrm{t}, J=8.2 \mathrm{~Hz}, 1 \mathrm{H})$, $7.45(\mathrm{~d}, J=7.7 \mathrm{~Hz}, 1 \mathrm{H}), 7.30(\mathrm{~d}, J=8.5 \mathrm{~Hz}, 2 \mathrm{H}), 2.41(\mathrm{~s}, 3 \mathrm{H}) ;{ }^{13} \mathrm{C}$ NMR $(101 \mathrm{MHz}, \mathrm{CDCl} 3) \delta 192.7$ $(\mathrm{d}, \mathrm{J}=3.7 \mathrm{~Hz}), 144.7,142.1,136.1,135.1,133.3(\mathrm{~d}, \mathrm{~J}=31.7 \mathrm{~Hz}), 123.0,127.3,124.7,122.0,120.47$ (d, $\mathrm{J}=6.3 \mathrm{~Hz}), 117.6,21.6 ;{ }^{19} \mathrm{~F}$ NMR $\left(376 \mathrm{MHz}, \mathrm{CDCl}_{3}\right) \delta-54.5$. HRMS (ESI) $\mathrm{m} / \mathrm{z}$ calcd for $\mathrm{C}_{15} \mathrm{H}_{12} \mathrm{~F}_{3} \mathrm{NO}_{3} \mathrm{~S}(\mathrm{MNa}+)$ 366.0382, found 366.0388. 


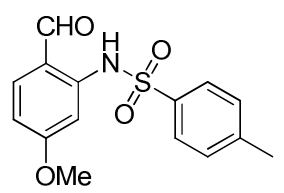

N-(2-formyl-5-methoxyphenyl)-4-methylbenzenesulfonamide (3fa) ${ }^{4}$

White solid, $28.1 \mathrm{mg}, 46 \%$ yield, mp: $145-147{ }^{\circ} \mathrm{C} .{ }^{1} \mathrm{H}$ NMR $\left(400 \mathrm{MHz}, \mathrm{CDCl}_{3}\right) \delta 11.13(\mathrm{~s}, 1 \mathrm{H}), 9.68$ (s, 1H), $7.80(\mathrm{~d}, J=8.3 \mathrm{~Hz}, 2 \mathrm{H}), 7.49(\mathrm{~d}, J=8.6 \mathrm{~Hz}, 1 \mathrm{H}), 7.28-7.26(\mathrm{~m}, 2 \mathrm{H}), 7.18(\mathrm{~d}, J=2.2 \mathrm{~Hz}, 1 \mathrm{H})$, $6.64(\mathrm{dd}, J=8.6,2.3 \mathrm{~Hz}, 1 \mathrm{H}), 3.86(\mathrm{~s}, 3 \mathrm{H}), 2.39(\mathrm{~s}, 3 \mathrm{H}) ;{ }^{13} \mathrm{C} \mathrm{NMR}\left(101 \mathrm{MHz}, \mathrm{CDCl}_{3}\right) \delta 193.2,165.5$, $144.3,142.4,138.2,136.3,129.8,127.3,115.9,109.4,102.4,55.8,21.6$.

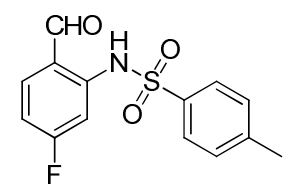

\section{N-(5-fluoro-2-formylphenyl)-4-methylbenzenesulfonamide (3ga) ${ }^{\mathbf{5}}$}

White solid, $34 \mathrm{mg}, 58 \%$ yield, mp: $123-125{ }^{\circ} \mathrm{C} .{ }^{1} \mathrm{H}$ NMR $\left(400 \mathrm{MHz}, \mathrm{CDCl}_{3}\right) \delta 11.07$ (s, 1H), $9.80(\mathrm{~s}$, $1 \mathrm{H}), 7.82(\mathrm{~d}, J=8.2 \mathrm{~Hz}, 2 \mathrm{H}), 7.62(\mathrm{dd}, J=8.5,6.2 \mathrm{~Hz}, 1 \mathrm{H}), 7.43(\mathrm{dd}, J=11.0,1.5 \mathrm{~Hz}, 1 \mathrm{H}), 7.30(\mathrm{~d}, J$ $=8.5 \mathrm{~Hz}, 2 \mathrm{H}), 6.85(\mathrm{dd}, J=5.3,4.5 \mathrm{~Hz}, 1 \mathrm{H}), 2.41(\mathrm{~s}, 3 \mathrm{H}) ;{ }^{13} \mathrm{C}$ NMR $\left(101 \mathrm{MHz}, \mathrm{CDCl}_{3}\right) \delta 193.6,167.0$ $(\mathrm{d}, J=258.1 \mathrm{~Hz}), 144.6,142.6(\mathrm{~d}, J=12.9 \mathrm{~Hz}), 138.8(\mathrm{~d}, J=11.9 \mathrm{~Hz}), 136.0,130.0,127.3,118.5$, $110.4(\mathrm{~d}, J=22.9 \mathrm{~Hz}), 105.0(\mathrm{~d}, J=27.9 \mathrm{~Hz}), 21.6 ;{ }^{19} \mathrm{~F} \mathrm{NMR}\left(376 \mathrm{MHz}, \mathrm{CDCl}_{3}\right) \delta-97.34(\mathrm{~s})$.<smiles>Cc1ccc(S(=O)(=O)Nc2cc(Cl)ccc2C=O)cc1</smiles>

N-(5-chloro-2-formylphenyl)-4-methylbenzenesulfonamide (3ha) ${ }^{4}$ White solid, $34.6 \mathrm{mg}, 56 \%$ yield, mp: $141-143{ }^{\circ} \mathrm{C} .{ }^{1} \mathrm{H}$ NMR $\left(400 \mathrm{MHz}, \mathrm{CDCl}_{3}\right) \delta 10.91(\mathrm{~s}, 1 \mathrm{H}), 9.81$ $(\mathrm{s}, 1 \mathrm{H}), 7.81(\mathrm{~d}, J=8.3 \mathrm{~Hz}, 2 \mathrm{H}), 7.73(\mathrm{~d}, J=1.5 \mathrm{~Hz}, 1 \mathrm{H}), 7.54(\mathrm{~d}, J=8.2 \mathrm{~Hz}, 1 \mathrm{H}), 7.30(\mathrm{~d}, J=8.3 \mathrm{~Hz}$, $2 \mathrm{H}), 7.14(\mathrm{dd}, J=8.2,1.8 \mathrm{~Hz}, 1 \mathrm{H}), 2.41(\mathrm{~s}, 3 \mathrm{H}) ;{ }^{13} \mathrm{C}$ NMR $\left(101 \mathrm{MHz}, \mathrm{CDCl}_{3}\right) \delta 193.9,144.6,142.6$, 141.0, 137.1, 136.0, 130.0, 127.3, 123.3, 120.1, 117.7, 21.6.<smiles>Cc1ccc(S(=O)(=O)Nc2cc(Br)ccc2C=O)cc1</smiles>

N-(5-bromo-2-formylphenyl)-4-methylbenzenesulfonamide (3ia) ${ }^{6}$

White solid, $39.5 \mathrm{mg}, 56 \%$ yield, mp: $145-147^{\circ} \mathrm{C} .{ }^{1} \mathrm{H}$ NMR (400 MHz, $\left.\mathrm{CDCl}_{3}\right) \delta 10.87$ (s, 1H), 9.80 $(\mathrm{s}, 1 \mathrm{H}), 7.90(\mathrm{~s}, 1 \mathrm{H}), 7.81(\mathrm{~d}, J=8.2 \mathrm{~Hz}, 2 \mathrm{H}), 7.46(\mathrm{~d}, J=8.2 \mathrm{~Hz}, 1 \mathrm{H}), 7.31-7.28(\mathrm{~m}, 3 \mathrm{H}), 2.41(\mathrm{~s}$, $3 \mathrm{H}) ;{ }^{13} \mathrm{C}$ NMR $\left(101 \mathrm{MHz}, \mathrm{CDCl}_{3}\right) \delta 194.2,144.6,140.8,137.0,136.0,131.5,130.0,127.3,126.3$, $120.7,120.4,21.6$.<smiles>Cc1ccc(S(=O)(=O)Nc2ccc(Br)cc2C=O)cc1</smiles>

N-(4-bromo-2-formylphenyl)-4-methylbenzenesulfonamide (3ja) ${ }^{7}$

Yellow solid, $46.6 \mathrm{mg}, 66 \%$ yield, mp: $132-134{ }^{\circ} \mathrm{C} .{ }^{1} \mathrm{H}$ NMR $\left(400 \mathrm{MHz}, \mathrm{CDCl}_{3}\right) \delta 10.91(\mathrm{~s}, 1 \mathrm{H}), 9.81$ $(\mathrm{s}, 1 \mathrm{H}), 7.81(\mathrm{~d}, J=8.1 \mathrm{~Hz}, 2 \mathrm{H}), 7.73(\mathrm{~s}, 1 \mathrm{H}), 7.54(\mathrm{~d}, J=8.2 \mathrm{~Hz}, 1 \mathrm{H}), 7.30(\mathrm{~d}, J=8.3 \mathrm{~Hz}, 2 \mathrm{H}), 7.15-$ $7.13(\mathrm{~m}, 1 \mathrm{H}), 2.41(\mathrm{~s}, 3 \mathrm{H}) ;{ }^{13} \mathrm{C}$ NMR $\left(101 \mathrm{MHz}, \mathrm{CDCl}_{3}\right) \delta 193.9,144.6,142.6,141.0,137.1,136.0$, $130.0,127.3,123.3,120.1,117.7,21.6$.<smiles>Cc1ccc(S(=O)(=O)Nc2ccccc2C=O)cc1</smiles>

N-(2-formylphenyl)-4-methylbenzenesulfonamide (3ka) ${ }^{8}$ 
Yellow solid, $28.6 \mathrm{mg}, 52 \%$ yield, mp: $172-174{ }^{\circ} \mathrm{C} .{ }^{1} \mathrm{H}$ NMR $\left(400 \mathrm{MHz}, \mathrm{CDCl}_{3}\right) \delta 10.81(\mathrm{~s}, 1 \mathrm{H})$, $9.85(\mathrm{~s}, 1 \mathrm{H}), 7.79(\mathrm{~d}, J=8.3 \mathrm{~Hz}, 2 \mathrm{H}), 7.71(\mathrm{~d}, J=8.4 \mathrm{~Hz}, 1 \mathrm{H}), 7.62-7.60(\mathrm{~m}, 1 \mathrm{H}), 7.55-7.51(\mathrm{~m}, 1 \mathrm{H})$, $7.26(\mathrm{~d}, J=8.4 \mathrm{~Hz}, 2 \mathrm{H}), 7.18(\mathrm{t}, J=7.5 \mathrm{~Hz}, 1 \mathrm{H}), 2.39(\mathrm{~s}, 3 \mathrm{H}) ;{ }^{13} \mathrm{C} \mathrm{NMR}\left(101 \mathrm{MHz}, \mathrm{CDCl}_{3}\right) \delta 195.0$, 144.2, 140.0, 136.4, 136.1, 135.8, 129.8, 127.3, 123.0, 121.9, 117.8, 21.6.

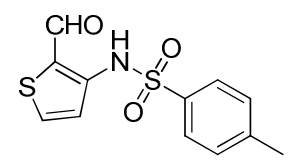

N-(2-formylthiophen-3-yl)-4-methylbenzenesulfonamide (3la) ${ }^{\mathbf{9}}$

Yellow solid, $28.1 \mathrm{mg}, 50 \%$ yield, mp: $110-111{ }^{\circ} \mathrm{C} .{ }^{1} \mathrm{H}$ NMR $\left(400 \mathrm{MHz}, \mathrm{CDCl}_{3}\right) \delta 10.17(\mathrm{~s}, 1 \mathrm{H})$, $9.61(\mathrm{~d}, J=0.6 \mathrm{~Hz}, 1 \mathrm{H}), 7.78(\mathrm{~d}, J=8.3 \mathrm{~Hz}, 2 \mathrm{H}), 7.64(\mathrm{~d}, J=5.3 \mathrm{~Hz}, 1 \mathrm{H}), 7.44(\mathrm{~d}, J=5.3 \mathrm{~Hz}, 1 \mathrm{H})$, $7.29(\mathrm{~d}, J=4.4 \mathrm{~Hz}, 2 \mathrm{H}), 2.41(\mathrm{~s}, 3 \mathrm{H}) ;{ }^{13} \mathrm{C} \mathrm{NMR}\left(101 \mathrm{MHz}, \mathrm{CDCl}_{3}\right) \delta 183.9,144.5,143.6,136.3,136.3$, 130.0, 127.1, $120.9,120.6,21.6$.

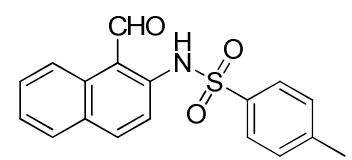

N-(1-formylnaphthalen-2-yl)-4-methylbenzenesulfonamide (3ma)

Black solid, $55.3 \mathrm{mg}, 85 \%$ yield, mp: $132-134{ }^{\circ} \mathrm{C} .{ }^{1} \mathrm{H}$ NMR $\left(400 \mathrm{MHz}, \mathrm{CDCl}_{3}\right) \delta 12.18(\mathrm{~s}, 1 \mathrm{H})$, $10.93(\mathrm{~s}, 1 \mathrm{H}), 8.40(\mathrm{~d}, J=8.7 \mathrm{~Hz}, 1 \mathrm{H}), 7.97(\mathrm{dd}, J=20.1,9.2 \mathrm{~Hz}, 2 \mathrm{H}), 7.83(\mathrm{dd}, J=8.0,3.4 \mathrm{~Hz}, 3 \mathrm{H})$, $7.65(\mathrm{t}, J=7.7 \mathrm{~Hz}, 1 \mathrm{H}), 7.49(\mathrm{t}, J=7.5 \mathrm{~Hz}, 1 \mathrm{H}), 7.26(\mathrm{~d}, J=8.1 \mathrm{~Hz}, 2 \mathrm{H}), 2.36(\mathrm{~s}, 3 \mathrm{H}) ;{ }^{13} \mathrm{C}$ NMR $(101$ $\left.\mathrm{MHz}, \mathrm{CDCl}_{3}\right) \delta 192.4,144.4,141.6,137.7,136.5,133.5,129.9,129.5,129.4,129.3,127.3,125.5$, 119.6, 116.8, 112.8, 21.6. HRMS (ESI) m/z calcd for $\mathrm{C}_{18} \mathrm{H}_{15} \mathrm{NO}_{3} \mathrm{~S}(\mathrm{MNa}+)$ 348.0665, found 348.0670.

\section{Experiments with isotopically-labeled substrates}

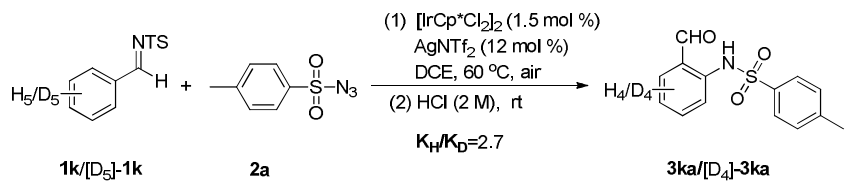

To a flask charged with N-Sulfonyl imine 1k or [D5]-1k $(0.24 \mathrm{mmol})$, sulfonyl azide $\mathbf{2 a}(0.2$ $\mathrm{mmol}$ ), [IrCp*Cl$]_{2}(2.4 \mathrm{mg}, 0.03 \mathrm{mmol}, 1.5 \mathrm{~mol} \%), \operatorname{AgNTf}_{2}(18.6 \mathrm{mg}, 0.48 \mathrm{mmol}, 12 \mathrm{~mol} \%)$, and 1,2-dichloroethane $(1.0 \mathrm{~mL})$. The reaction mixture was stirred at $60^{\circ} \mathrm{C}$ for $3 \mathrm{~h}$. After cooled to room temperature, $2 \mathrm{M} \mathrm{HCl}(1 \mathrm{~mL})$ was added. The resulting mixture was stirred at room temperature for another $3 \mathrm{~h}$ and then extracted with ethyl acetate $(3 \times 10 \mathrm{~mL})$. The solvents were removed under reduced pressure, and the residue was purified by chromatography on silica gel (eluent: petroleum ether/ethyl acetate $=10: 1$ ) to give the desired product $22.3 \mathrm{mg}$ of 3ka and 8.4 $\mathrm{mg}$ of [D4]-3ka. The ratio of $\mathrm{k}_{\mathrm{H}} / \mathrm{k}_{\mathrm{D}}$ was determined by the product yield of $\mathbf{3 k a}$ and [ $\left.\mathbf{D}_{\mathbf{4}}\right]-\mathbf{3 k a}$.

\section{Reference:}

1. Yokosaka, T.; Nemoto, T.; Nakayama, H.; Shiga, N.; Hamada, Y. Chem. Commun. 2014, 50, 12775.

2. Zhang, X.; Song, X.; Li, H.; Zhang, S.; Chen, X.; Yu, X.; Wang, W. Angew. Chem. Int. Ed. 2012, 51, 7282.

3. Yokosaka, T.; Shiga, N.; Nemoto, T. J. Org. Chem. 2014, 79. 3866

4. Dhiman, S.; Ramasastry, S. S. V. Org. Lett. 2015, 17, 5116.

5. Zhang, J.; Shao, J.; Xue, J.; Wang, Y.; Li, Y. RSC. Adv. 2014, 4, 63850.

6. Kang, K.T.; Kim, S.G. Synthesis, 2014, 40, 31.

7. Schammel, A. W.; Chiou, G.; Garg, N. K. Org . Lett. 2012, 14, 4556.

8. Nada, T.; Yoneshige, Y.; Ii, Y. Catalysis. 2016, 6, 3168.

9. Yang, T.; Zhang, T.; Yang, S.; Chen, S.; Li, X. Org. Biomol. Chem. 2014, 12, 4290. 


\section{NMR spectra of products}

\section{${ }^{1} \mathrm{H}$ NMR spectrum of $\mathbf{3 a a}$}

$\begin{array}{lll}1 & 1 \\ \frac{1}{1} & 0 & 0\end{array}$
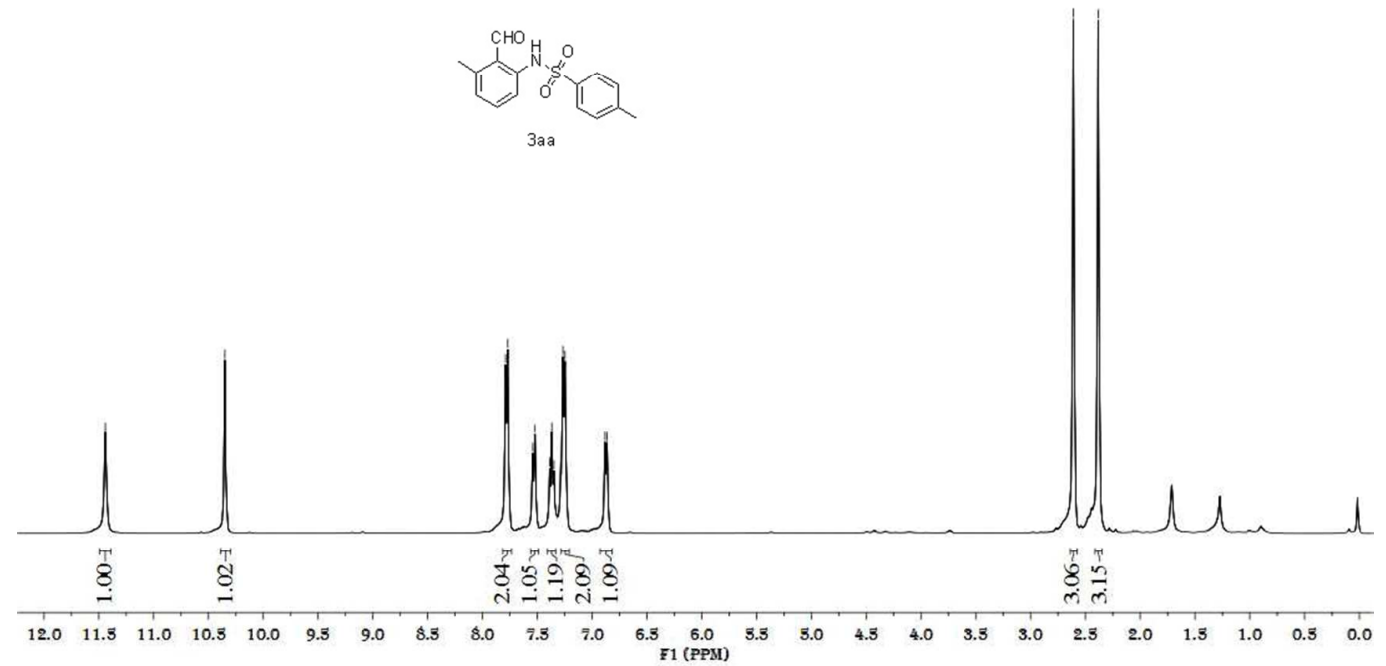

${ }^{13} \mathrm{C}$ NMR spectrum of $\mathbf{3 a a}$
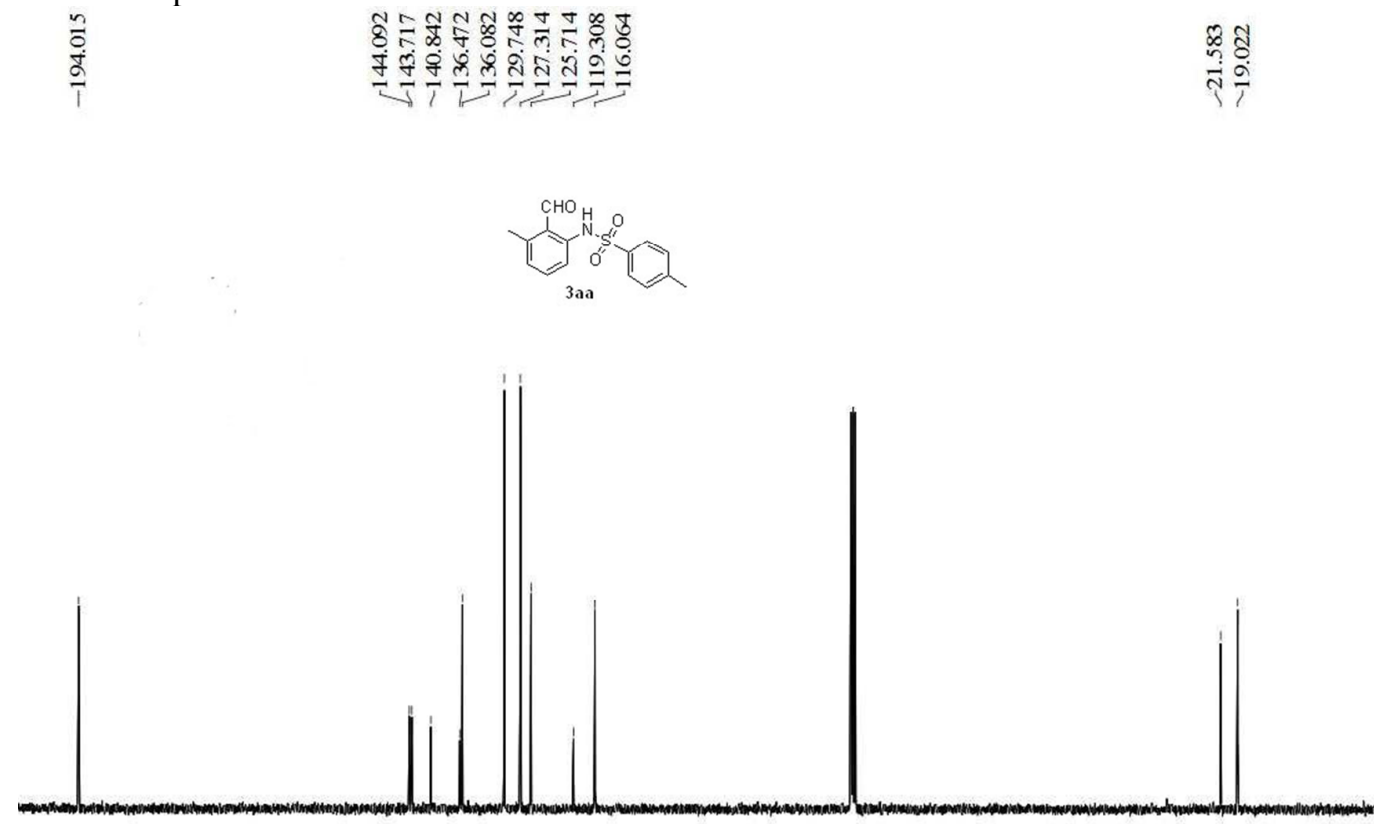

200

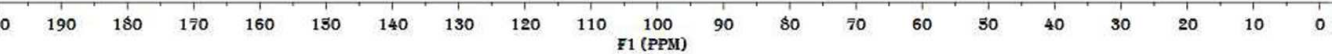


${ }^{1} \mathrm{H}$ NMR spectrum of $\mathbf{3 a b}$

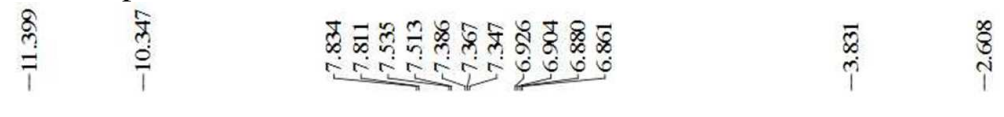

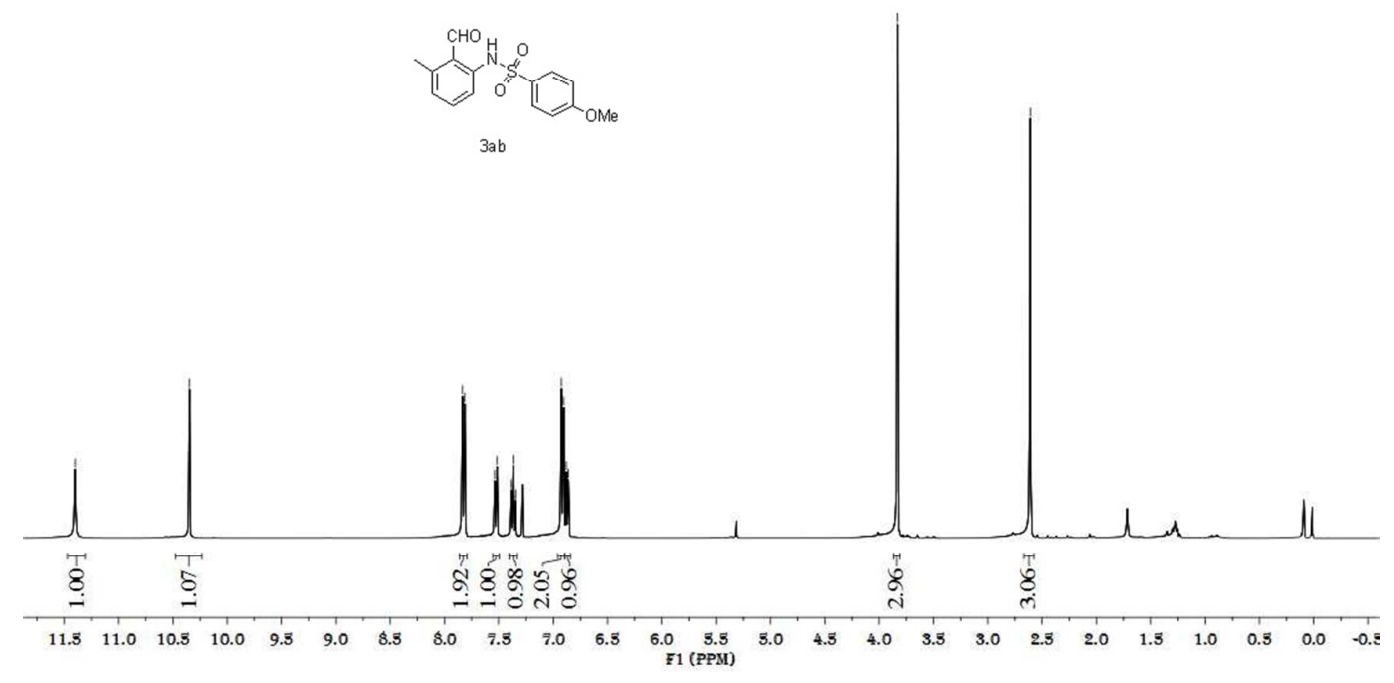

${ }^{13} \mathrm{C}$ NMR spectrum of $\mathbf{3 a b}$
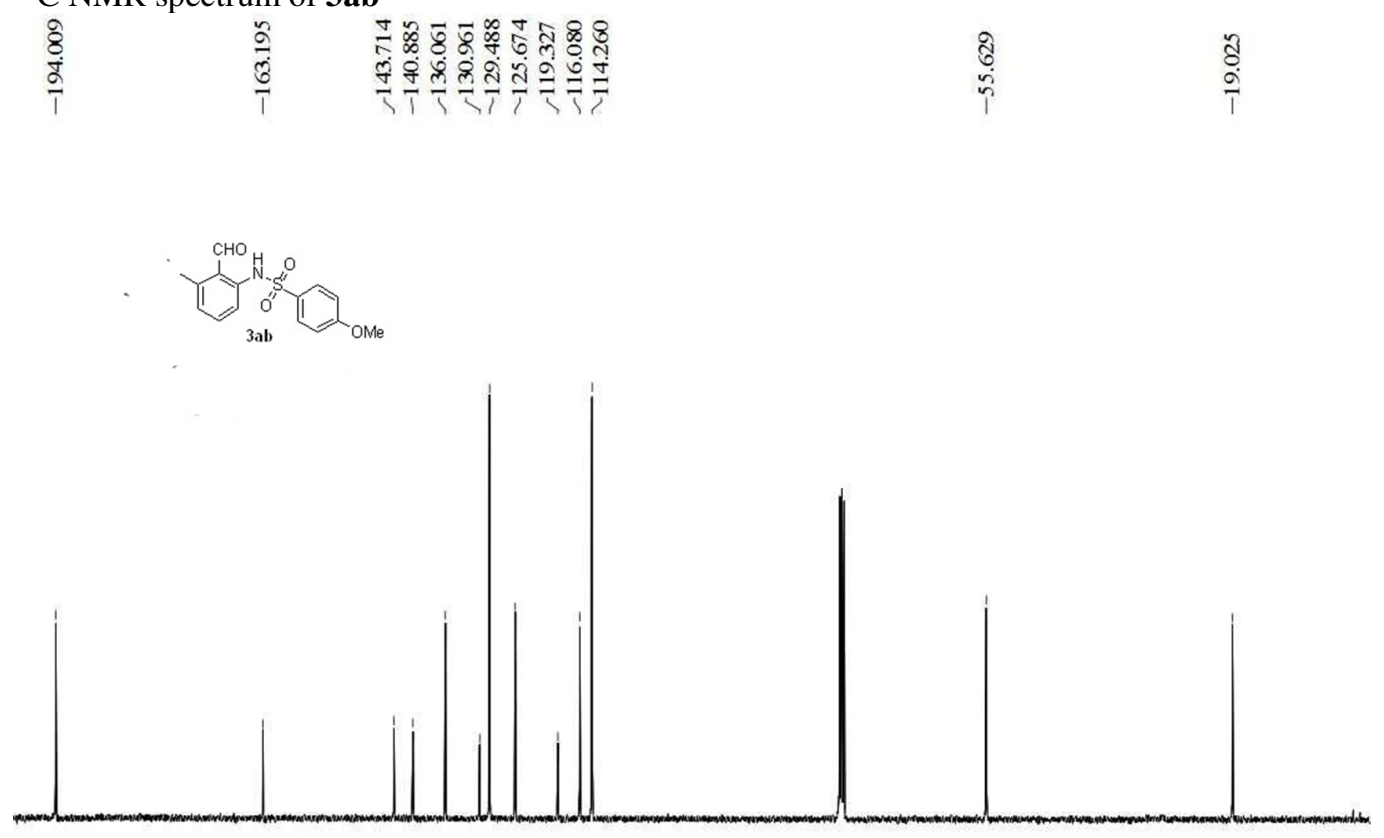

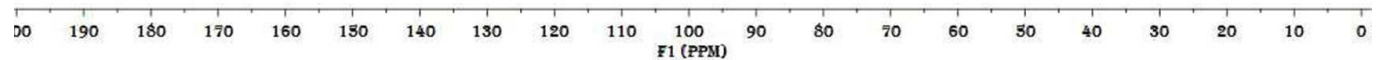




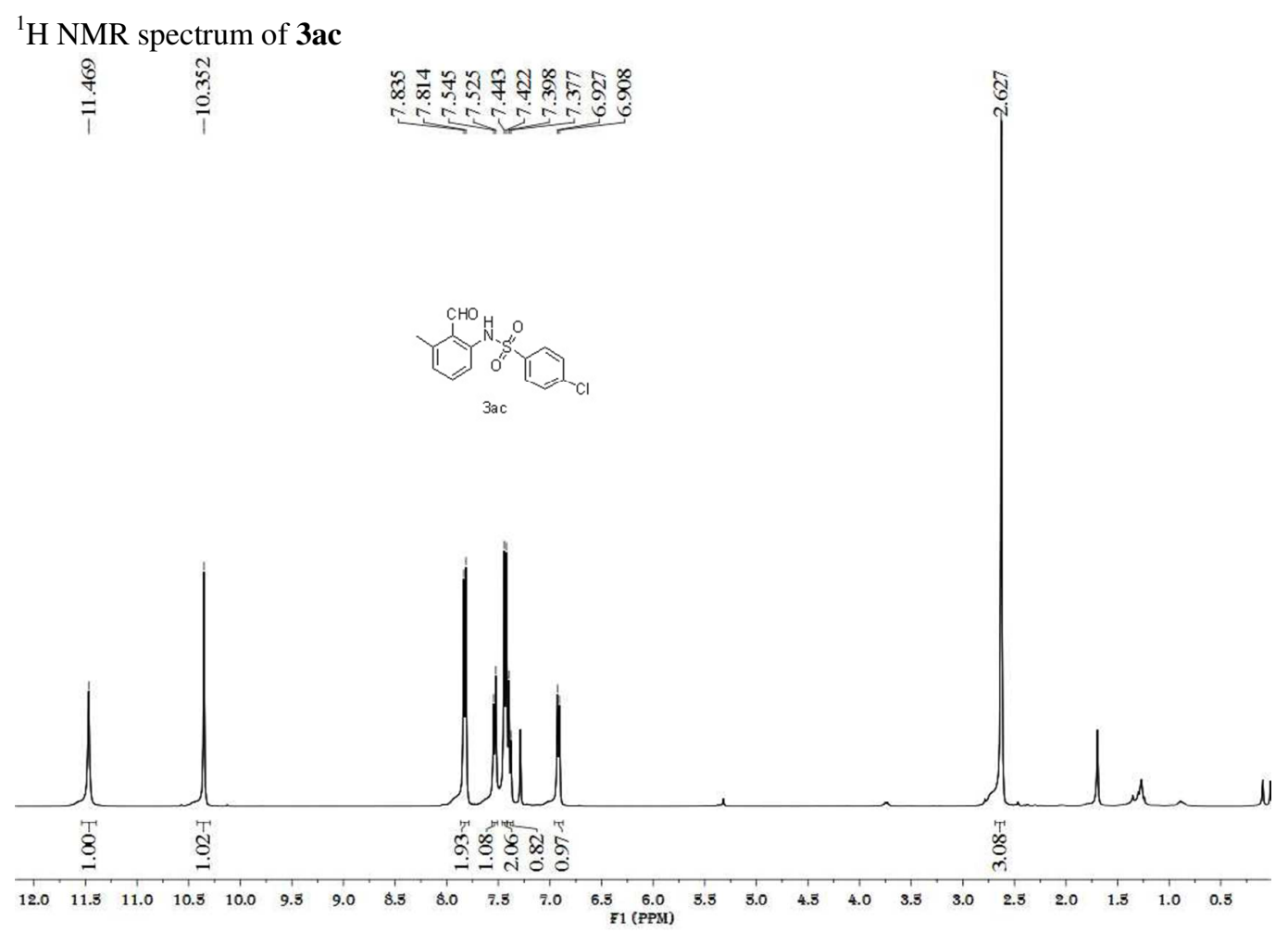

${ }^{13} \mathrm{C}$ NMR spectrum of $\mathbf{3 a c}$

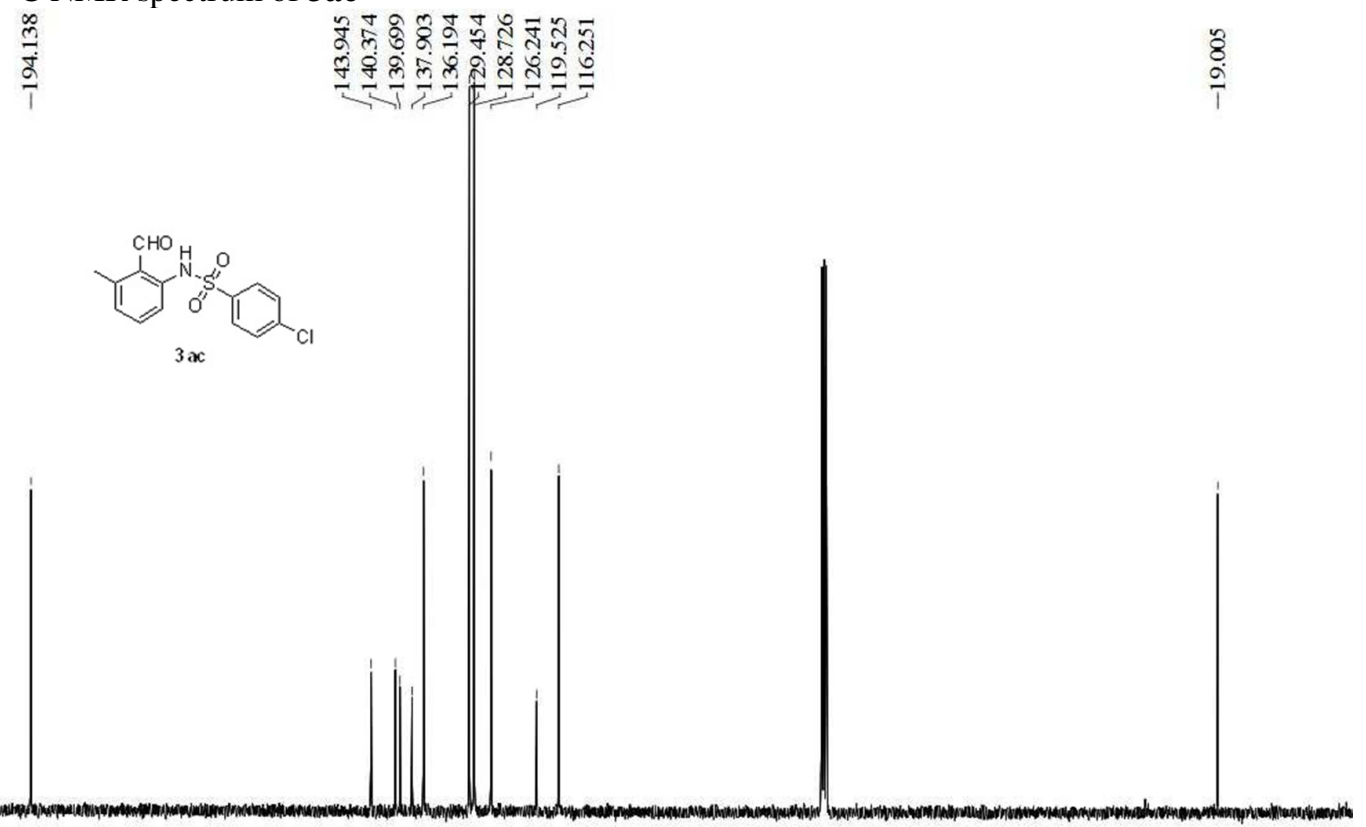

$\begin{array}{llllllllllllllllllllll}190 & 180 & 170 & 160 & 130 & 140 & 130 & 120 & 110 & 100 & 90 & 80 & 70 & 60 & 30 & 40 & 30 & 20 & 10 & 0\end{array}$ 
${ }^{1} \mathrm{H}$ NMR spectrum of $\mathbf{3 a d}$
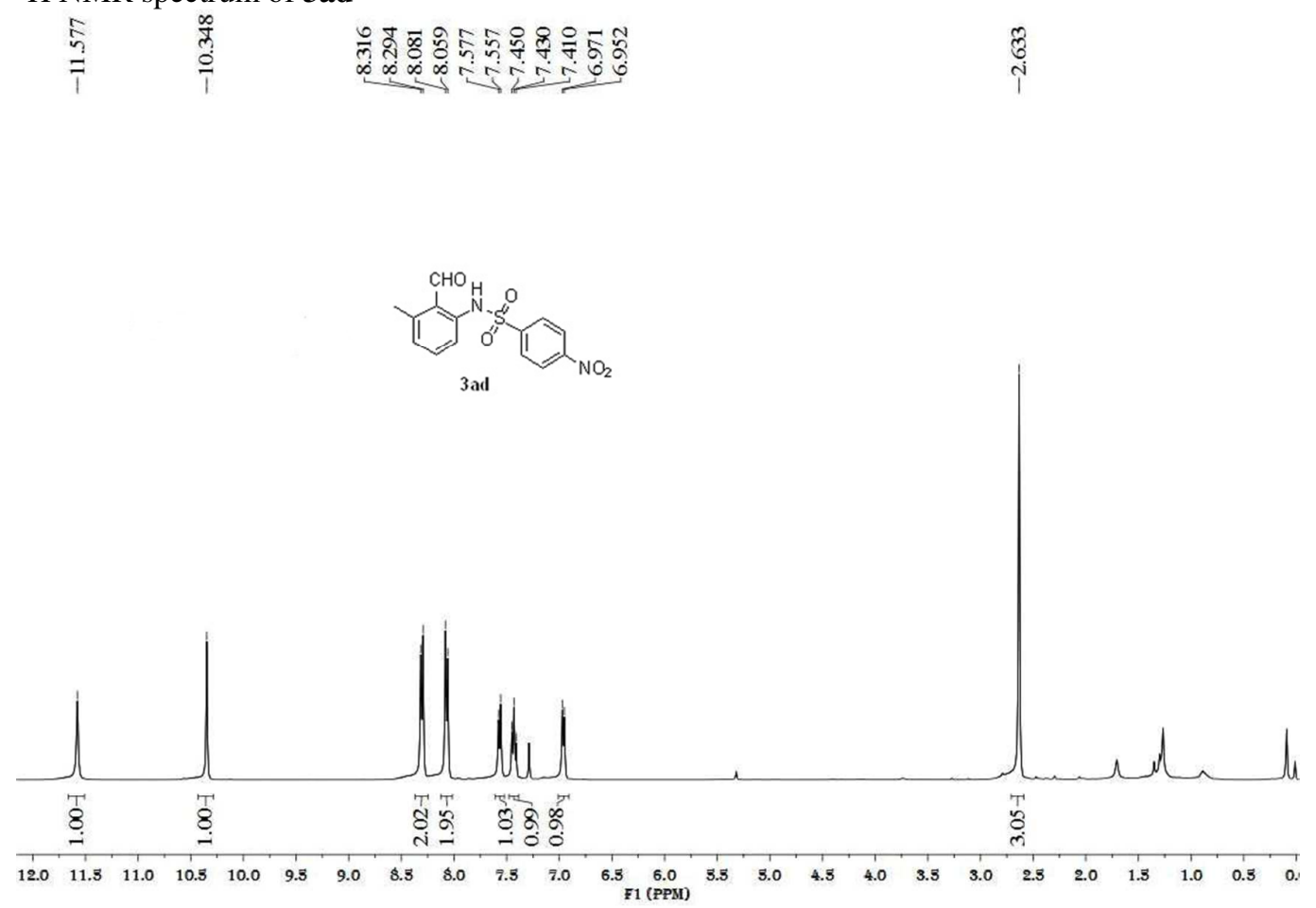

${ }^{13} \mathrm{C}$ NMR spectrum of $\mathbf{3 a d}$

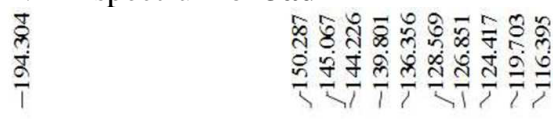

8
5
$\infty$
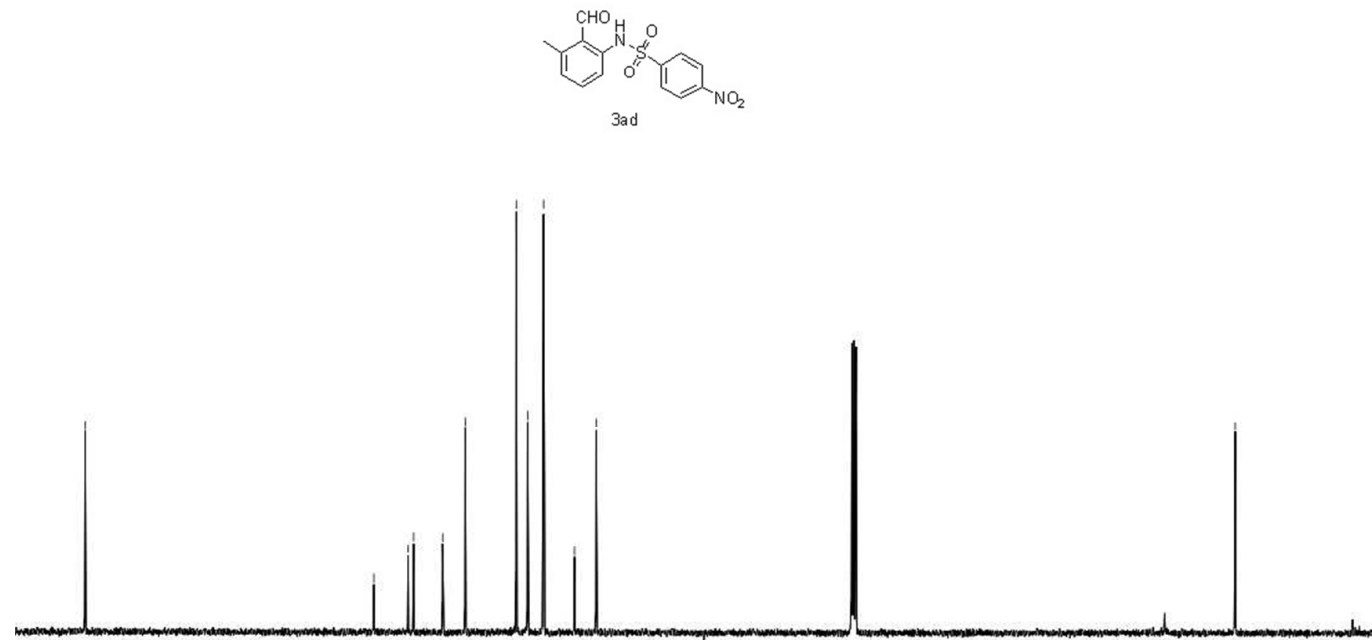

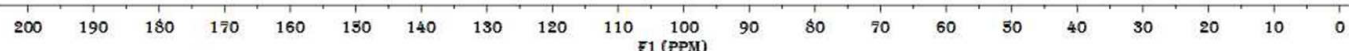


${ }^{1} \mathrm{H}$ NMR spectrum of $\mathbf{3 a e}$

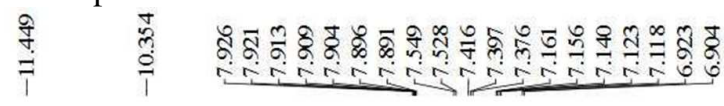
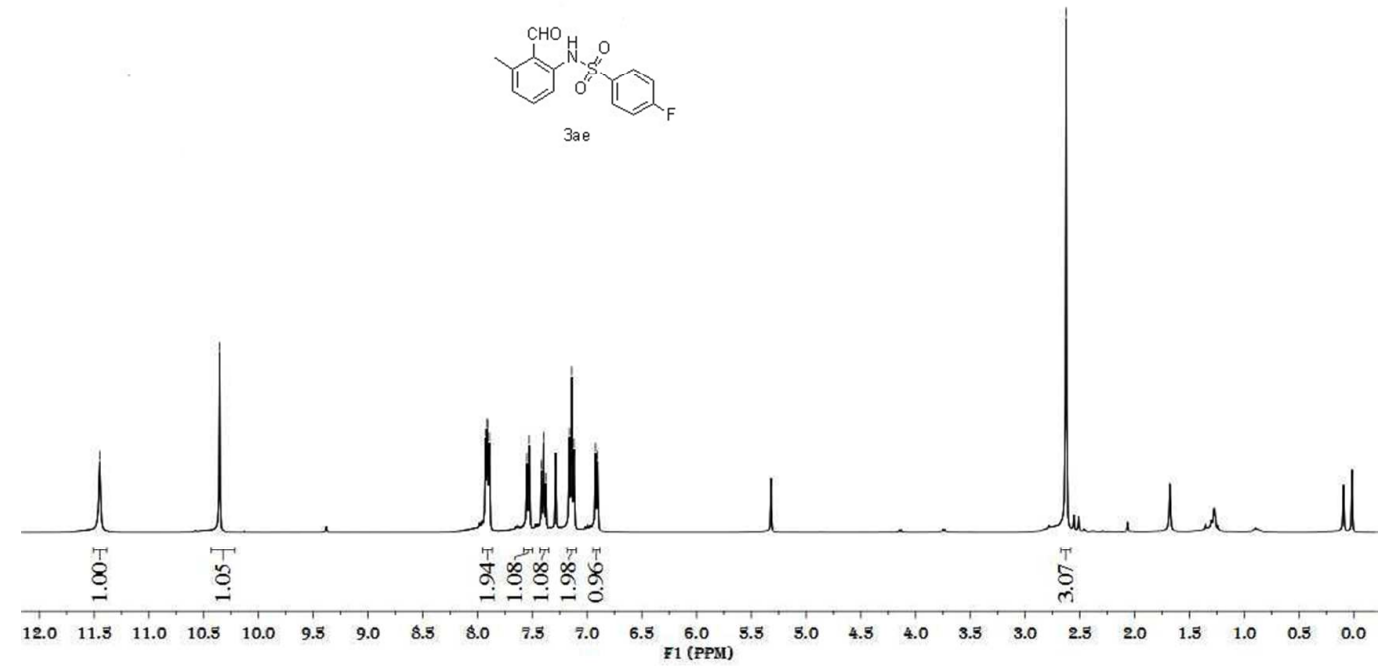

${ }^{13} \mathrm{C}$ NMR spectrum of $\mathbf{3 a e}$
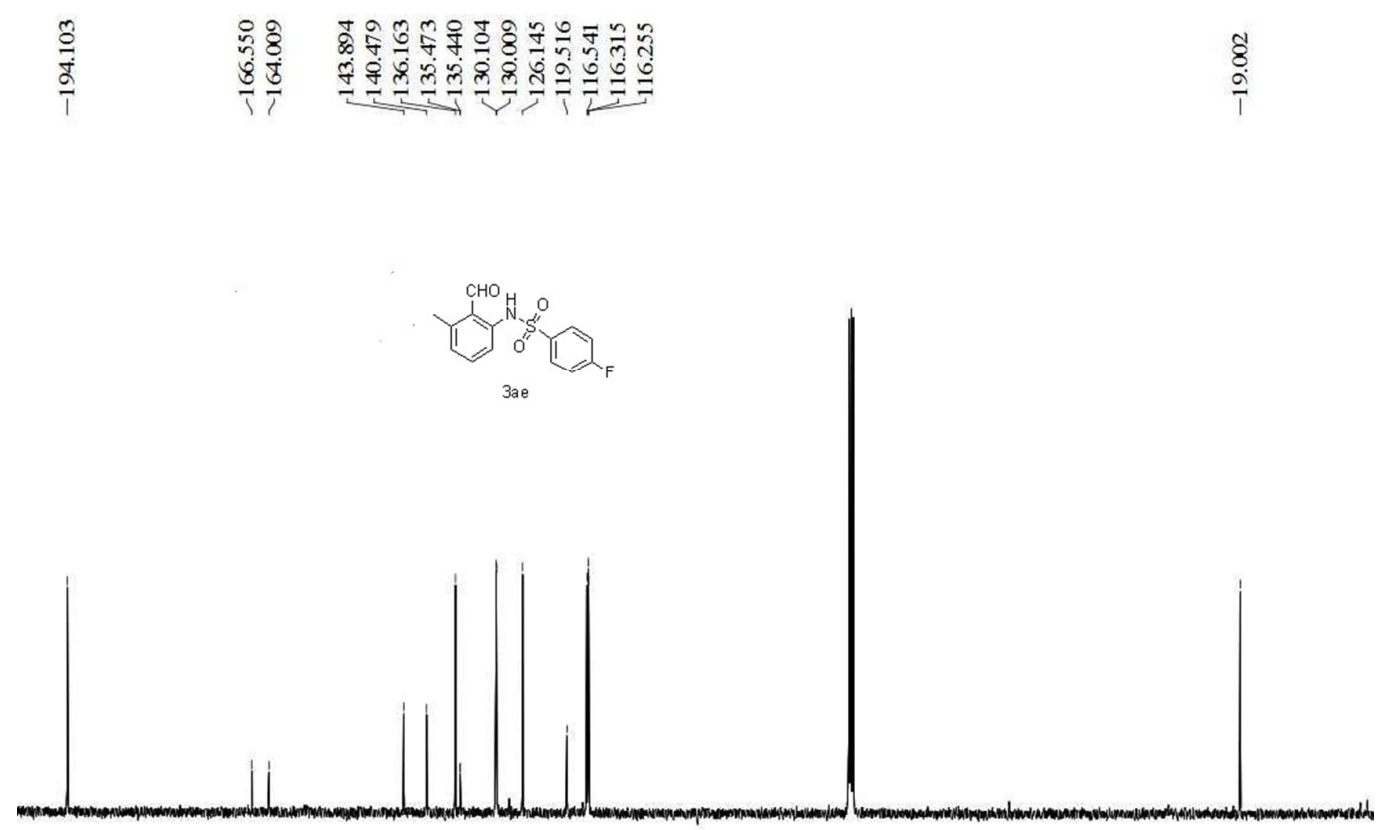

$200190 \quad 180 \quad 170 \quad 160 \quad 150 \quad 140 \quad 130 \quad 120 \quad 110 \begin{array}{lllllllllllllllllllll}100 & 90 & 80 & 70 & 60 & 50 & 40 & 30 & 20 & 10 & 0\end{array}$ 
${ }^{1} \mathrm{H}$ NMR spectrum of $\mathbf{3 a f}$

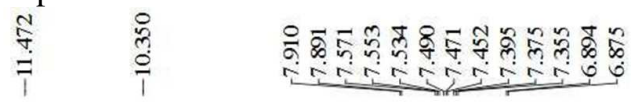

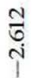

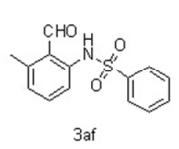

3af

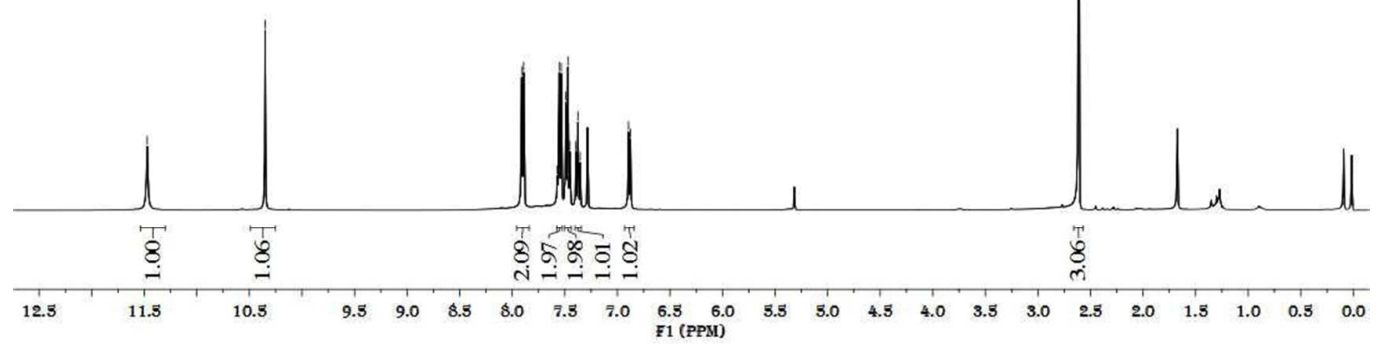

${ }^{13} \mathrm{C}$ NMR spectrum of $\mathbf{3 a f}$

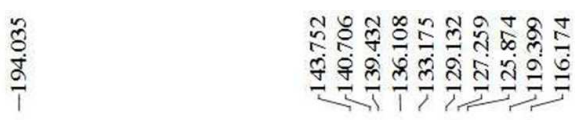

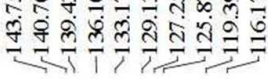

$\frac{8}{8}$

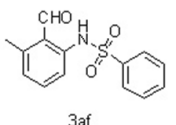

3af

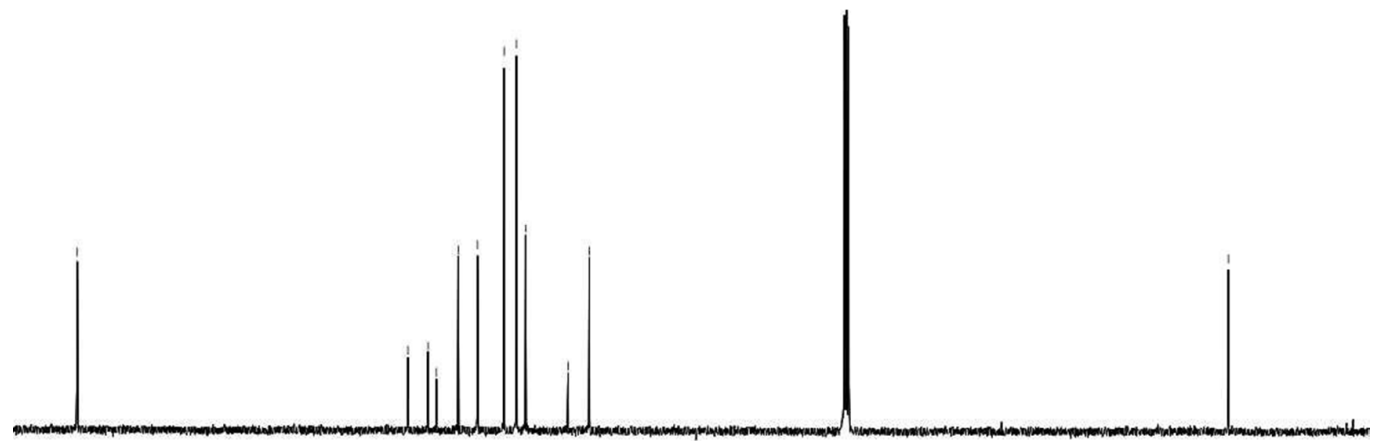

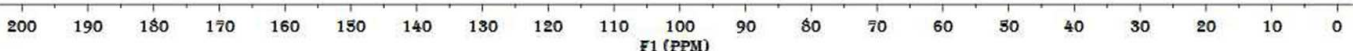


${ }^{1} \mathrm{H}$ NMR spectrum of $\mathbf{3 a g}$

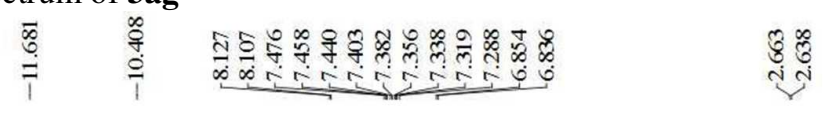
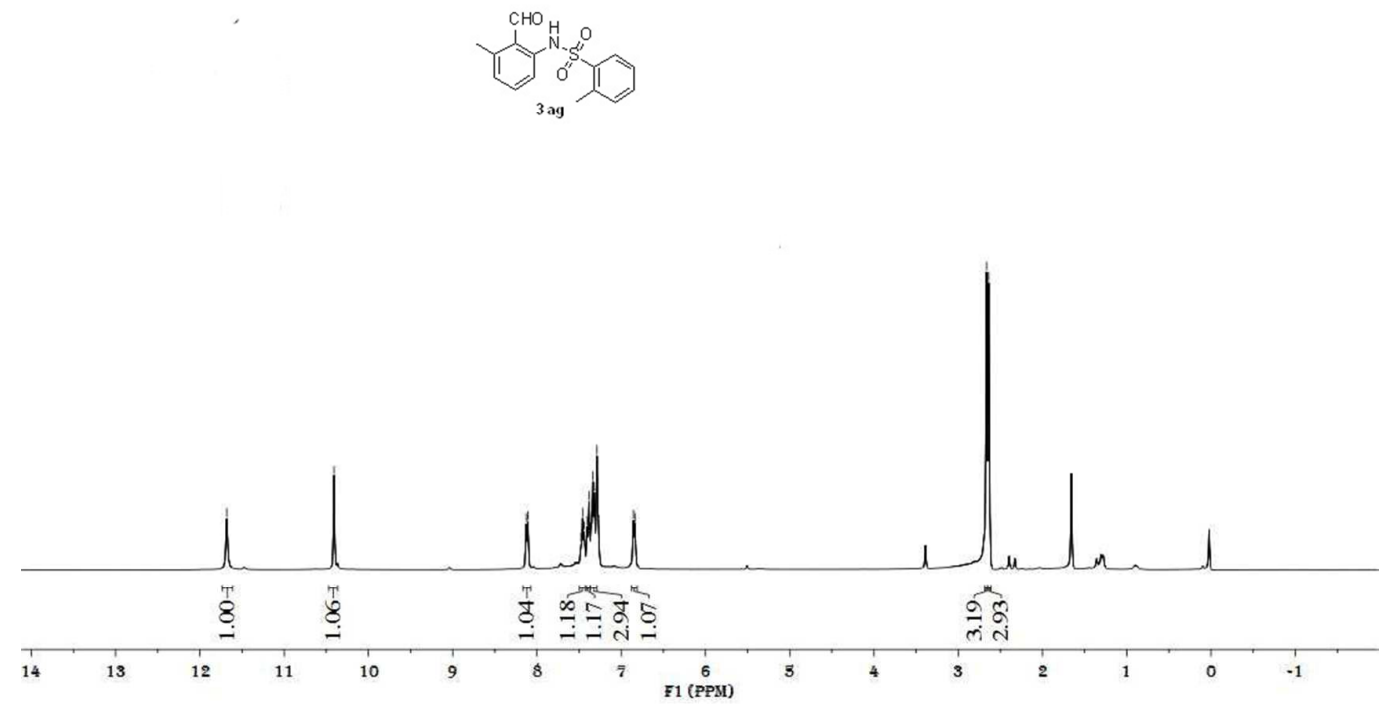

${ }^{13} \mathrm{C}$ NMR spectrum of $\mathbf{3 a g}$

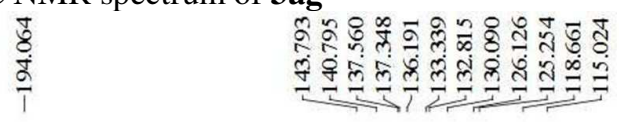

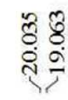

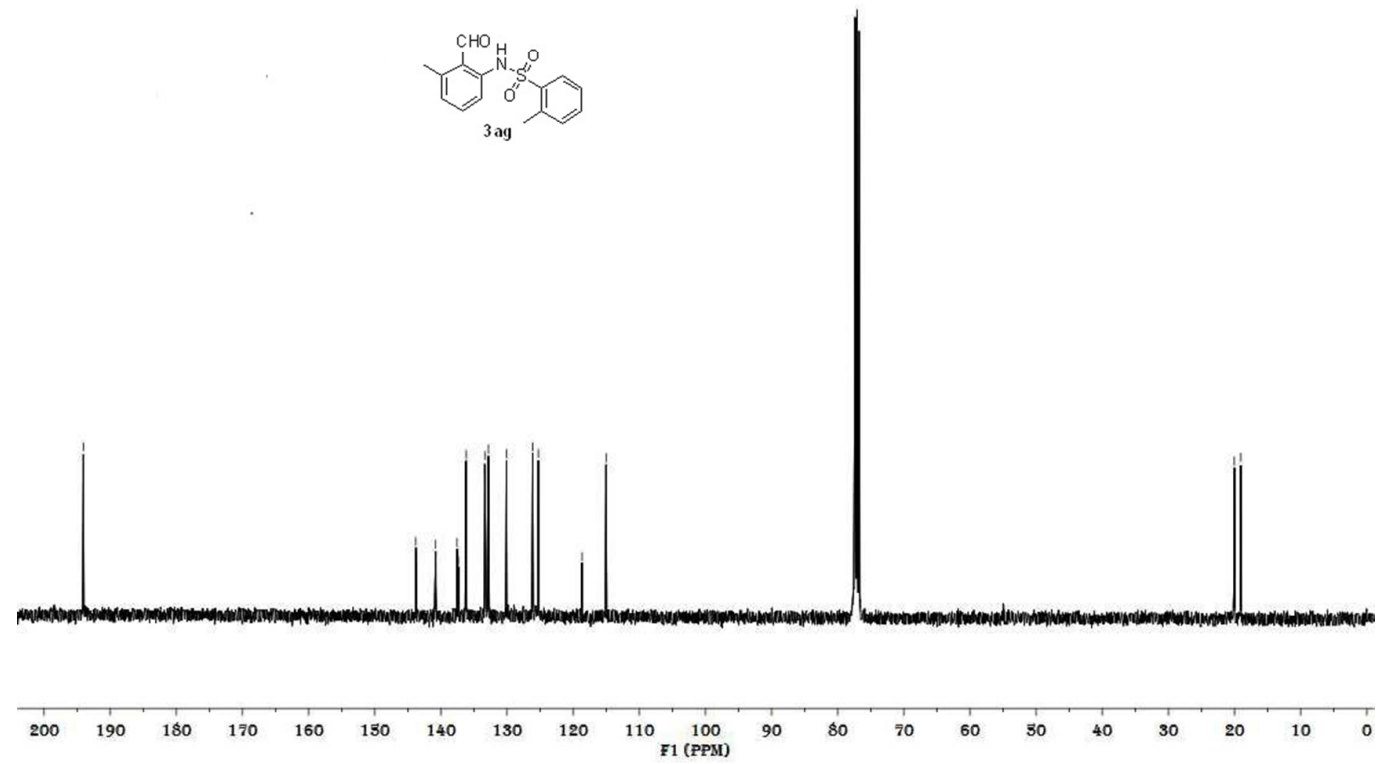


${ }^{1} \mathrm{H}$ NMR spectrum of $\mathbf{3 a h}$

1
$\cdots$
2
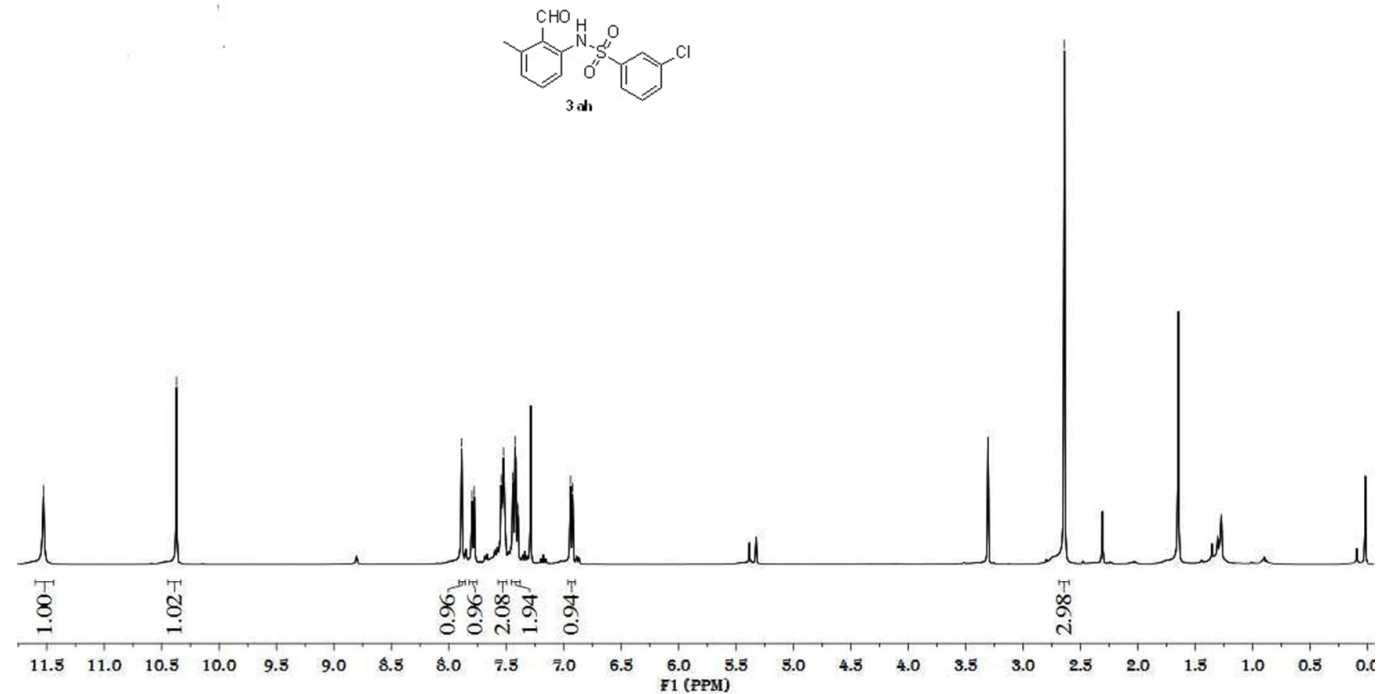

${ }^{13} \mathrm{C}$ NMR spectrum of $\mathbf{3 a h}$
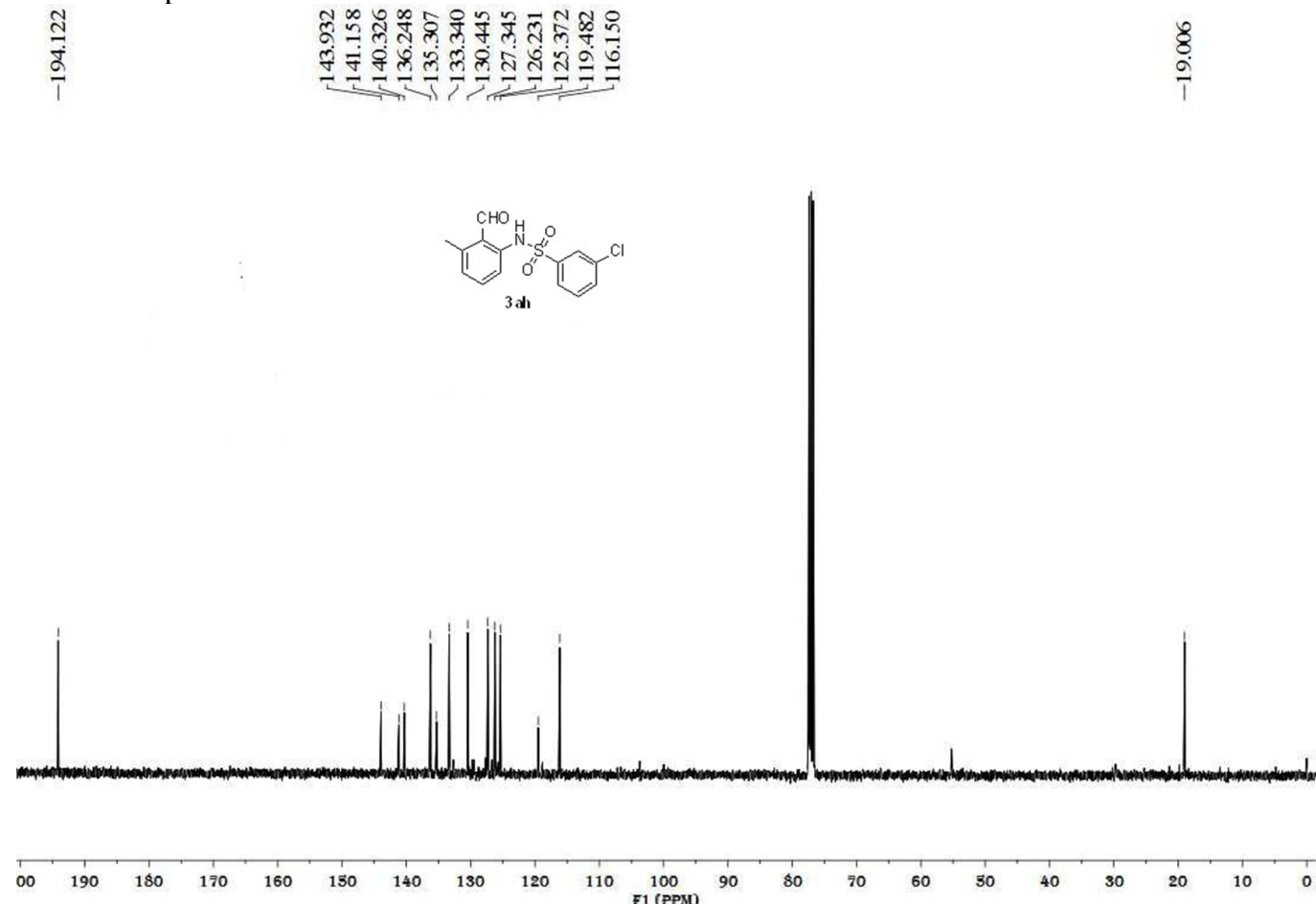
${ }^{1} \mathrm{H}$ NMR spectrum of 3ai

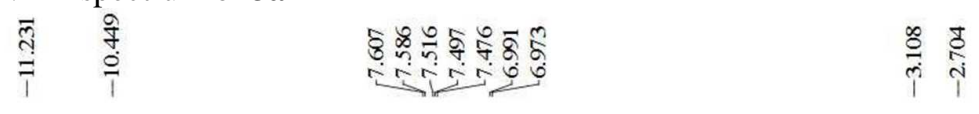

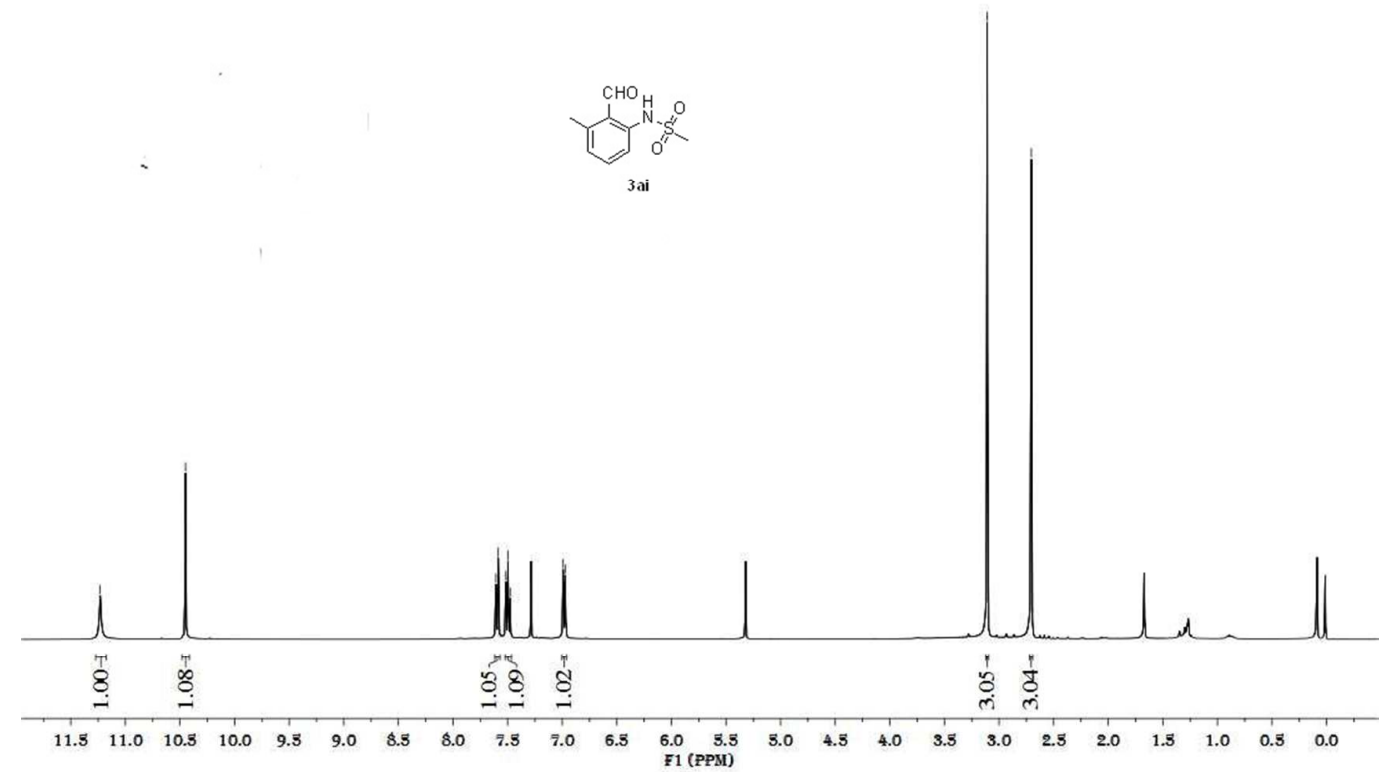

${ }^{13} \mathrm{C}$ NMR spectrum of 3ai

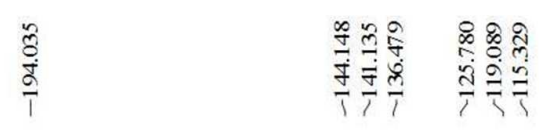

ঙั่

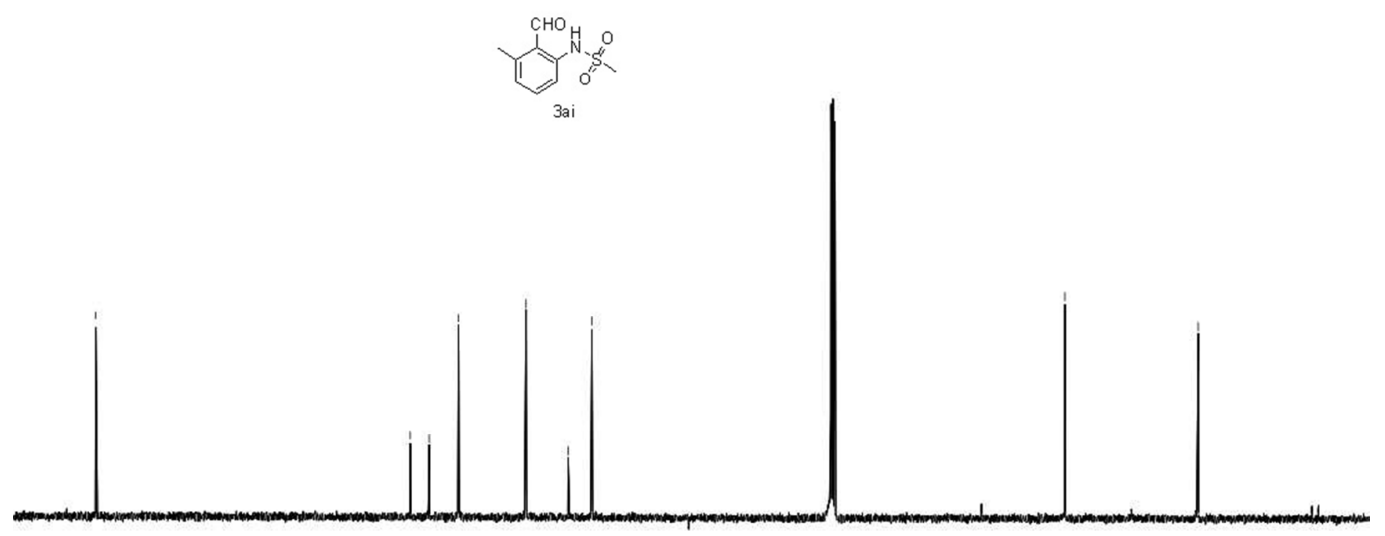

$200190 \quad 180 \quad 170 \quad 160 \quad 130 \quad 140 \quad 130 \quad 120 \quad 110 \begin{array}{llllllllllll}100 & 90 & 80 & 70 & 60 & 30 & 40 & 30 & 20 & 10 & 0\end{array}$ 
${ }^{1} \mathrm{H}$ NMR spectrum of $\mathbf{3 b a}$

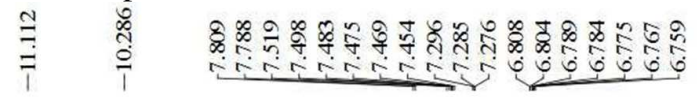

i

N

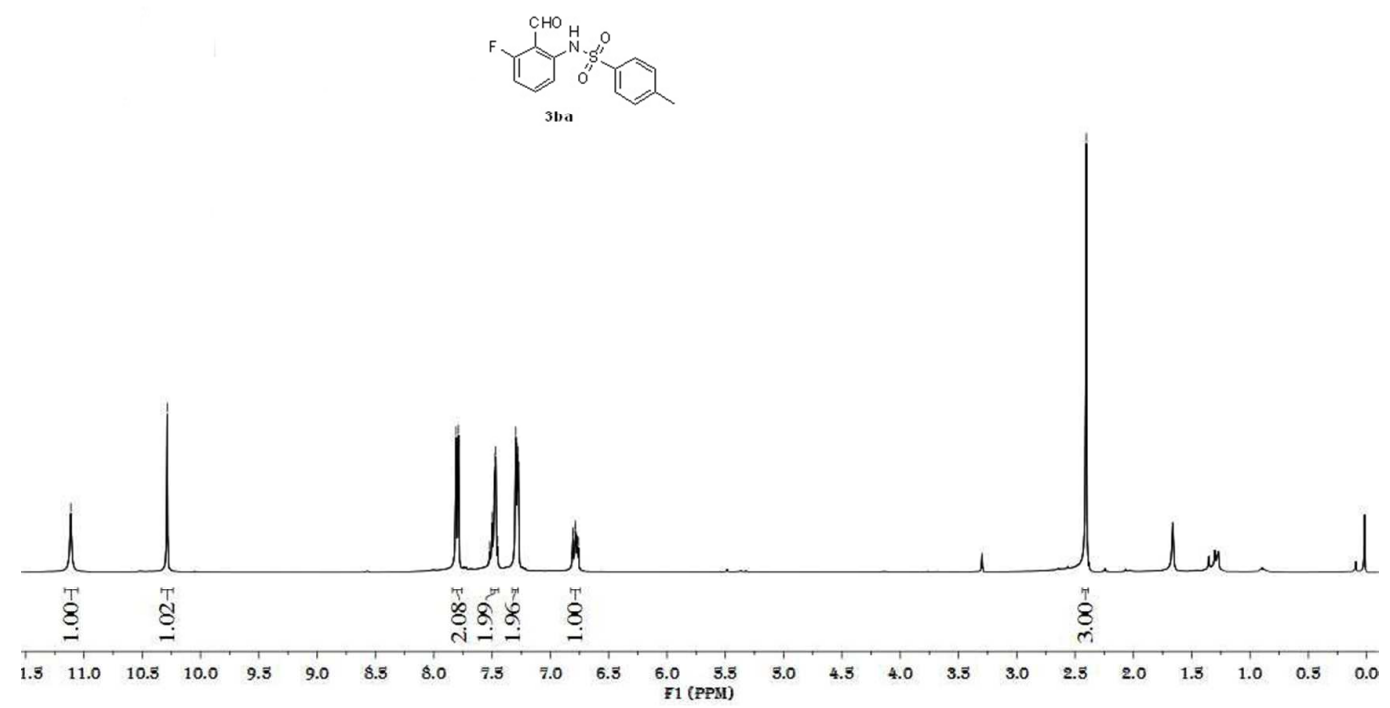

${ }^{13} \mathrm{C}$ NMR spectrum of $\mathbf{3 b a}$

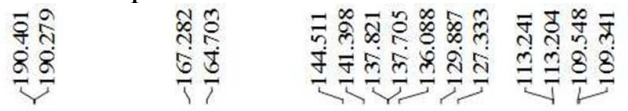

$\frac{N}{\frac{N}{1}}$
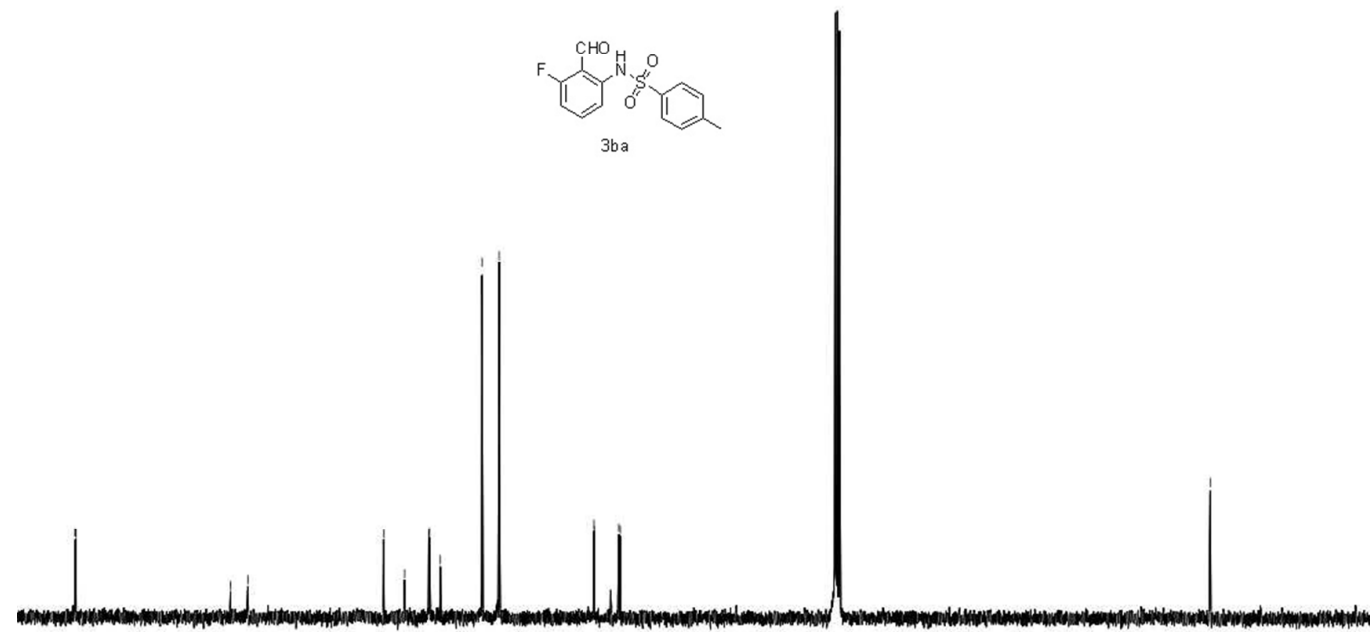

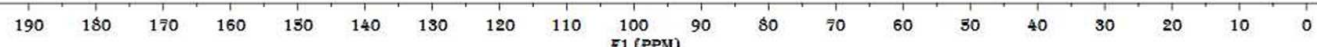


${ }^{1} \mathrm{H}$ NMR spectrum of $\mathbf{3 c a}$

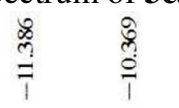

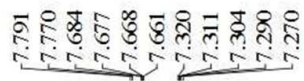

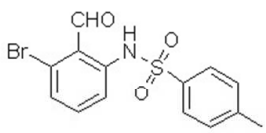

$3 c a$

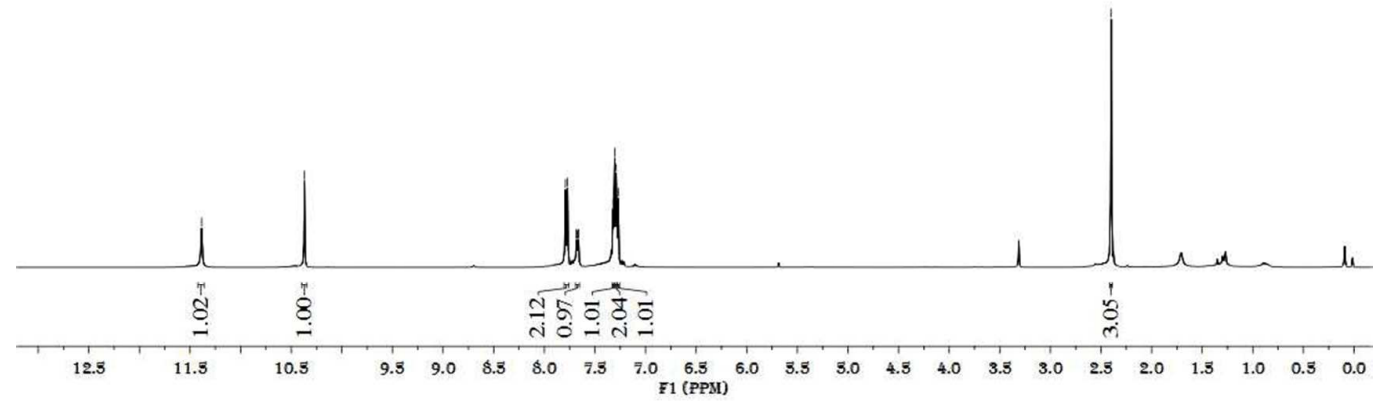

${ }^{13} \mathrm{C}$ NMR spectrum of $\mathbf{3 c a}$

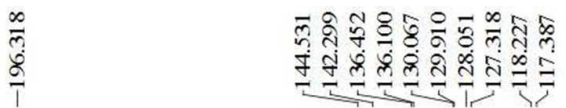

$\frac{\pi}{\frac{\pi}{1}}$

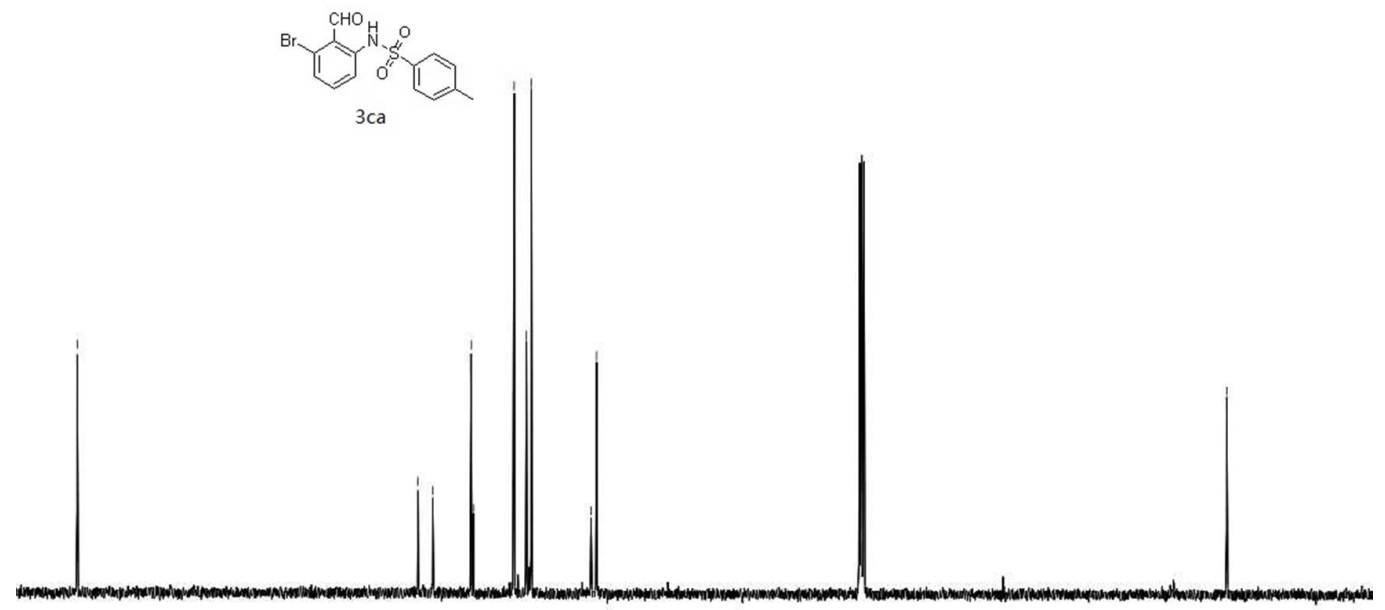
$\begin{array}{lllllllllll}200 & 190 & 180 & 170 & 160 & 130 & 140 & 130 & 120 & 110 & 100 \\ \text { F1 (PPSI) }\end{array}$ 
${ }^{1} \mathrm{H}$ NMR spectrum of $\mathbf{3 d a}$

$$
\begin{array}{lll}
2 & 0 \\
3 & 0 & 0
\end{array}
$$
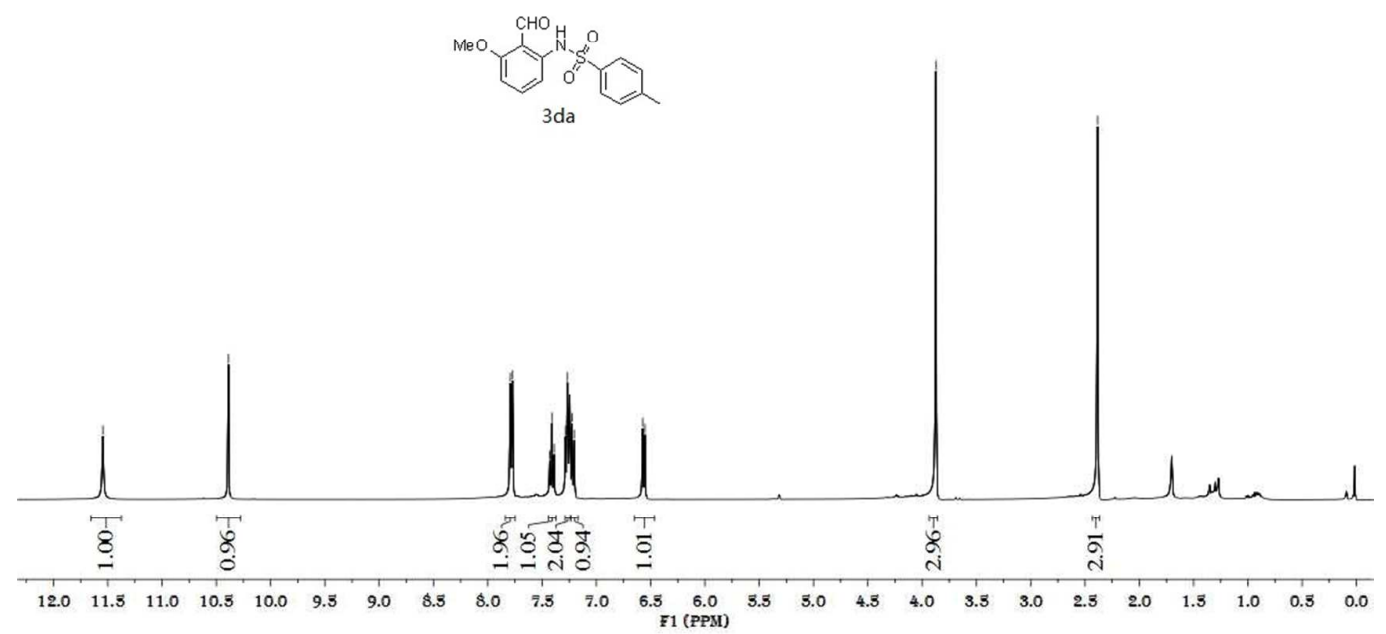

${ }^{13} \mathrm{C}$ NMR spectrum of $\mathbf{3 d a}$
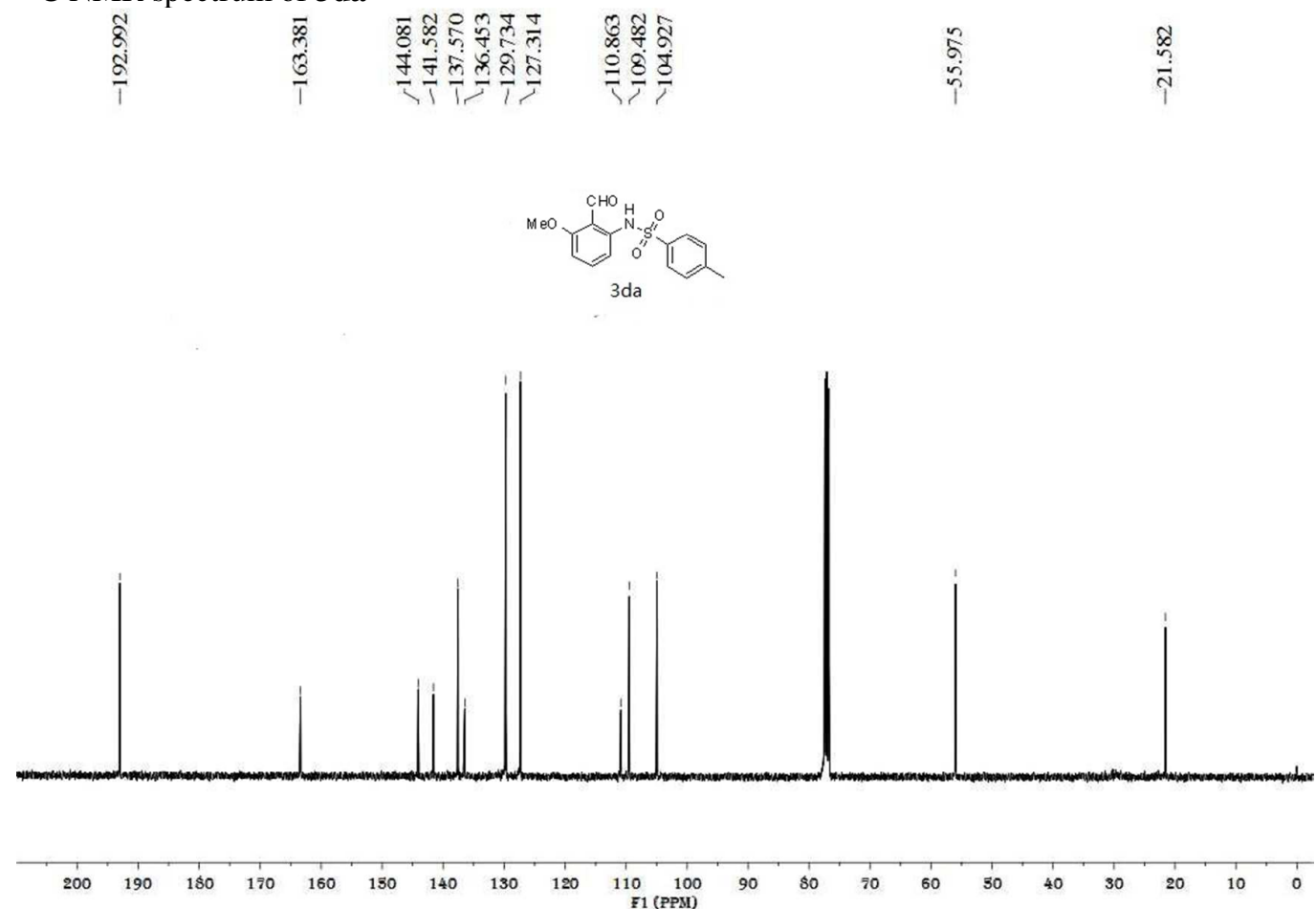
${ }^{1} \mathrm{H}$ NMR spectrum of $\mathbf{3 e a}$

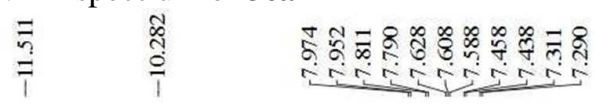

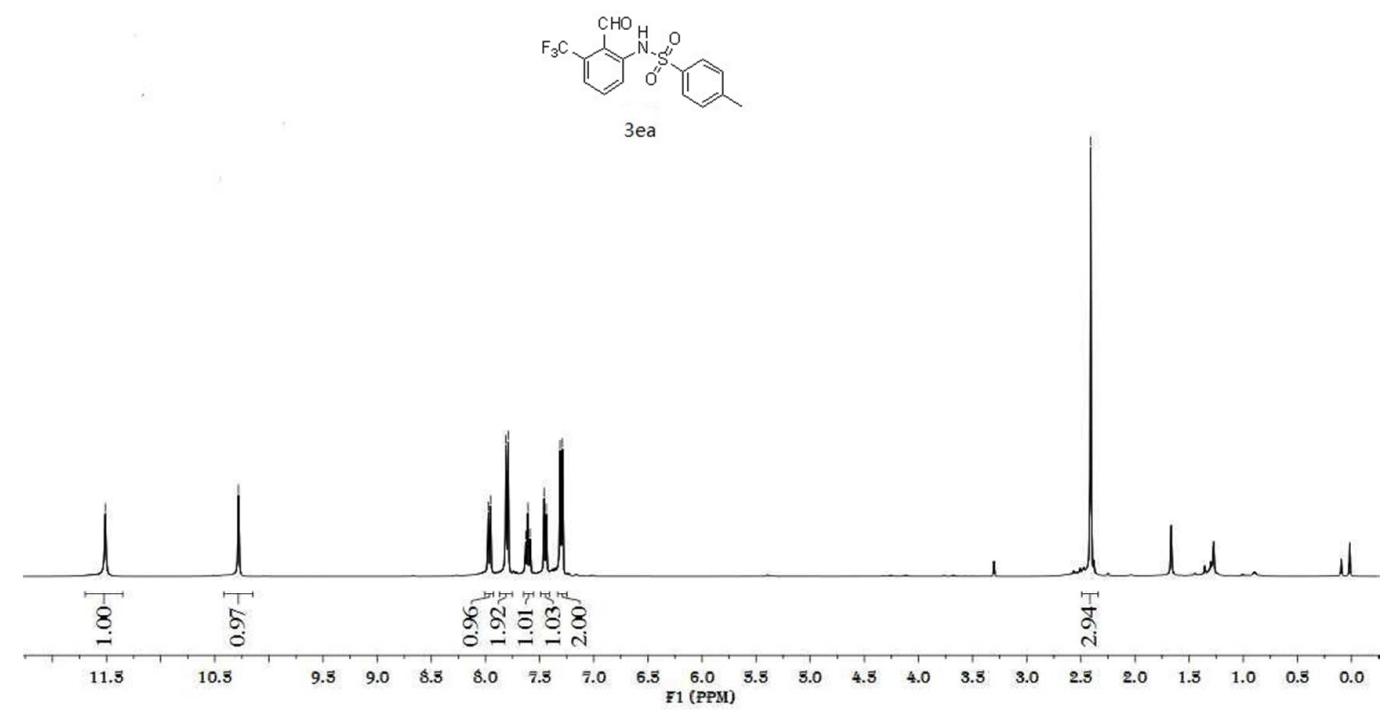

${ }^{13} \mathrm{C}$ NMR spectrum of $\mathbf{3 e a}$
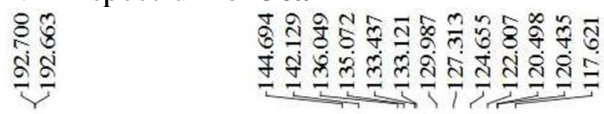

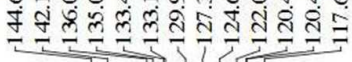
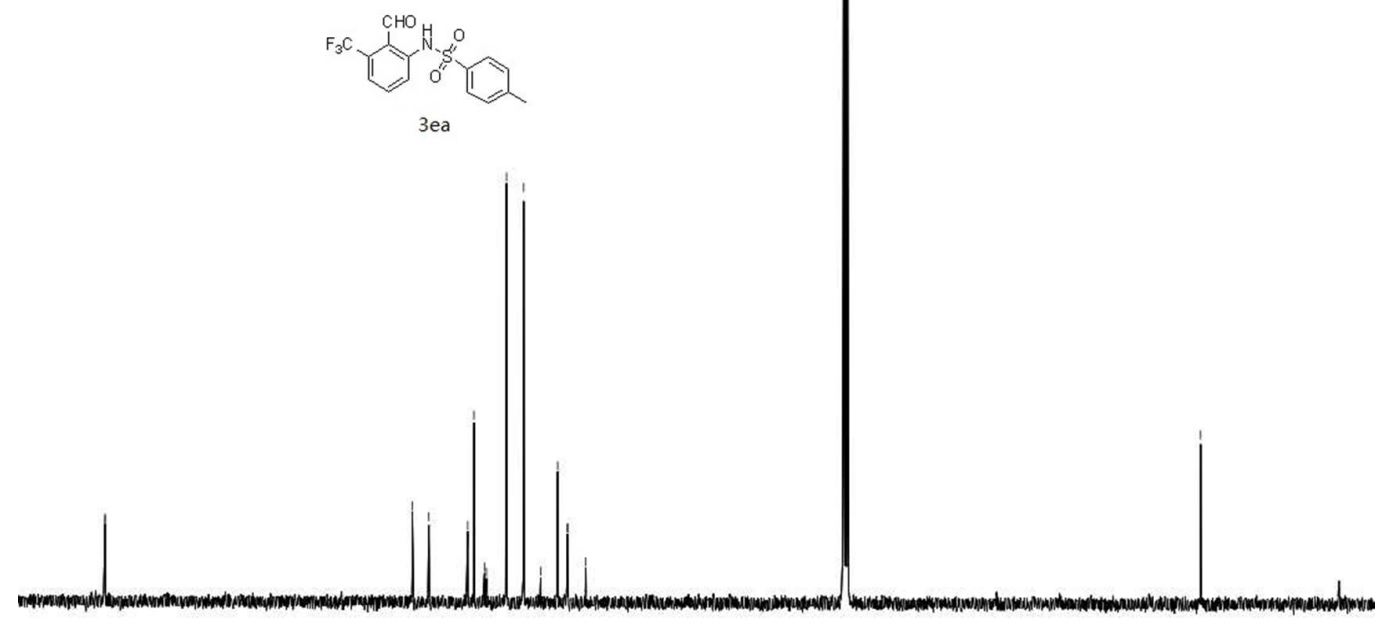

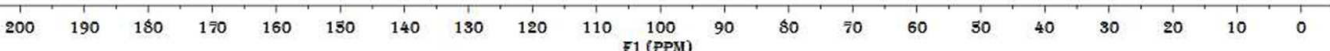


${ }^{1} \mathrm{H}$ NMR spectrum of $\mathbf{3 f a}$

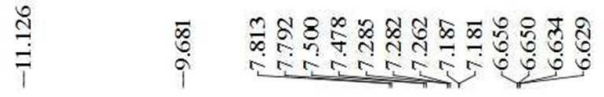

त)
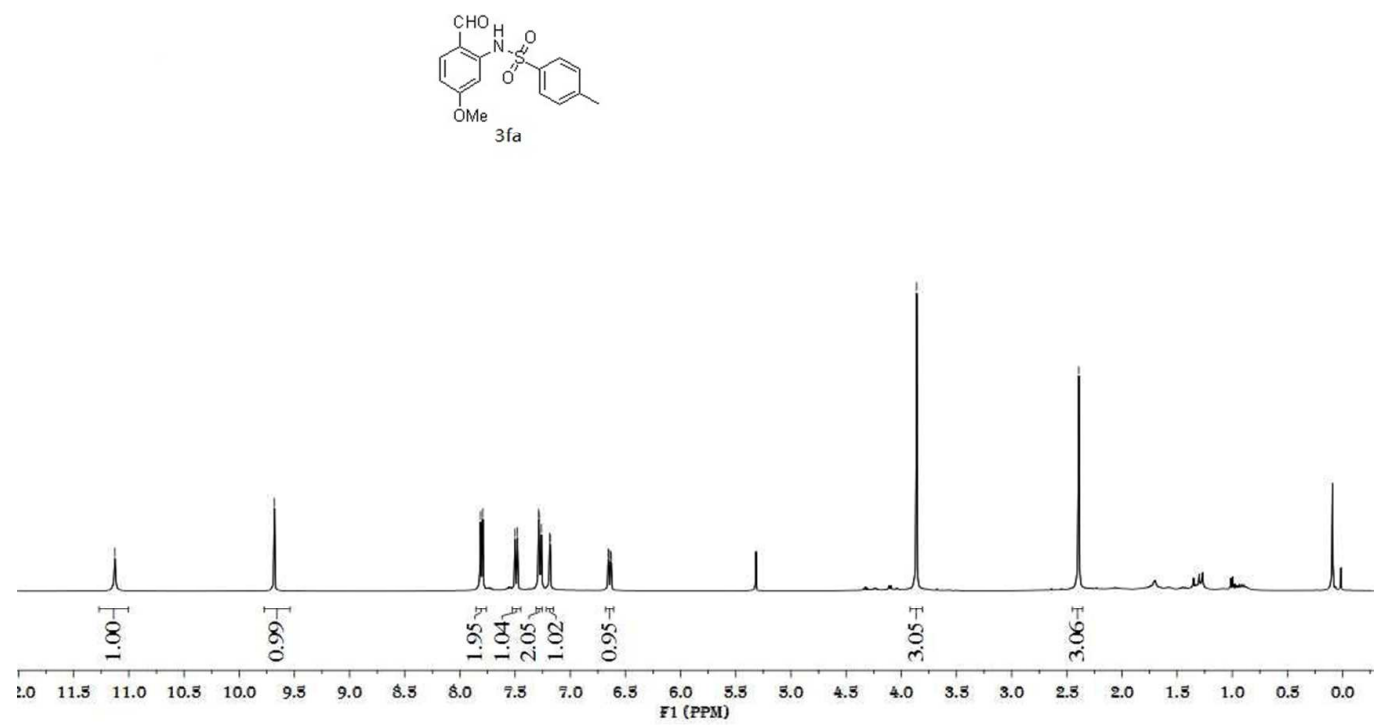

${ }^{13} \mathrm{C}$ NMR spectrum of $\mathbf{3 f a}$
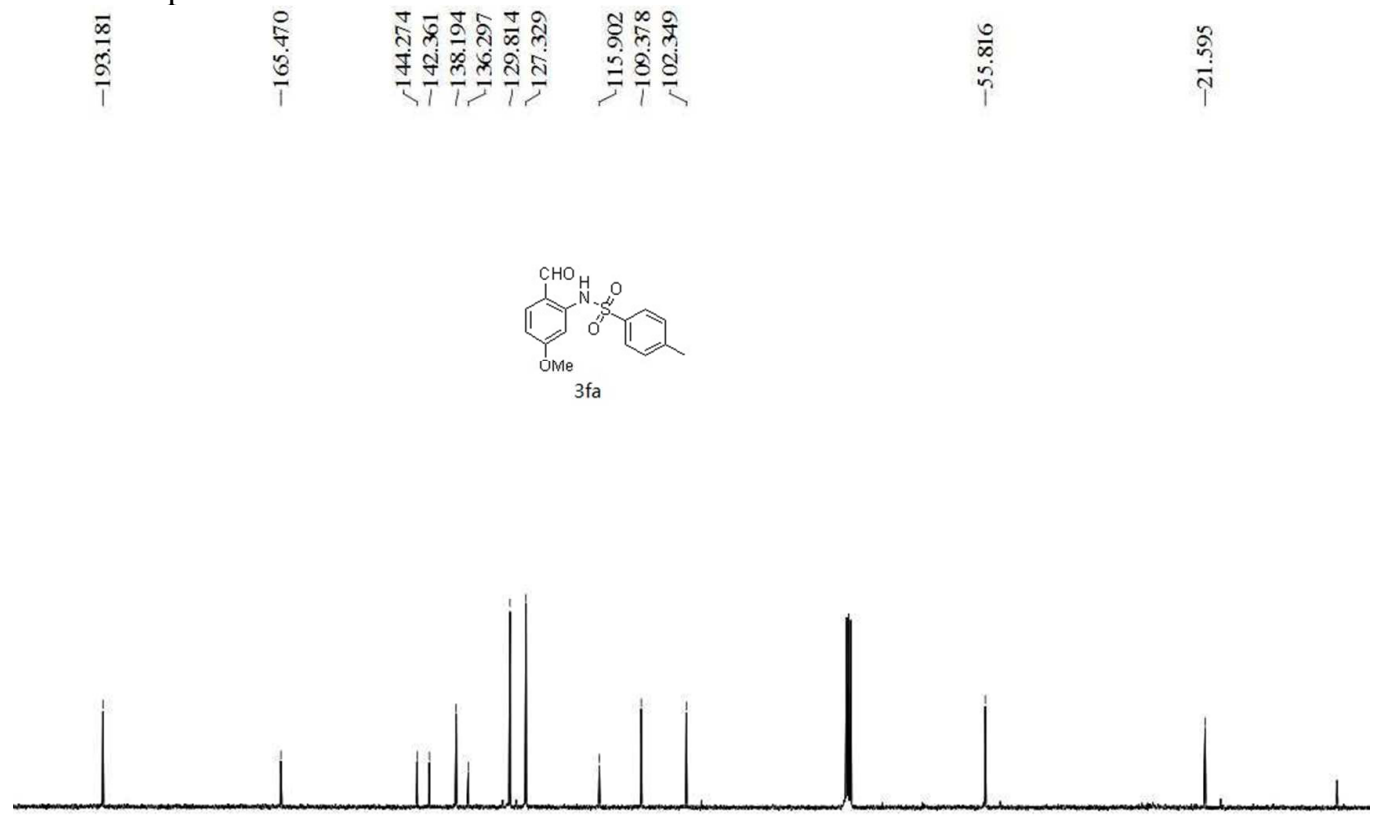

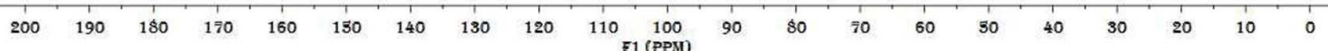


${ }^{1} \mathrm{H}$ NMR spectrum of $\mathbf{3 g a}$

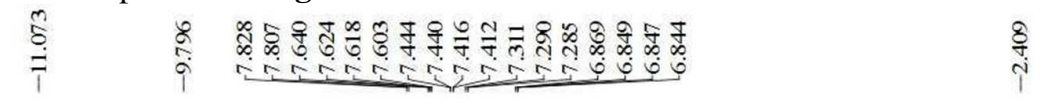
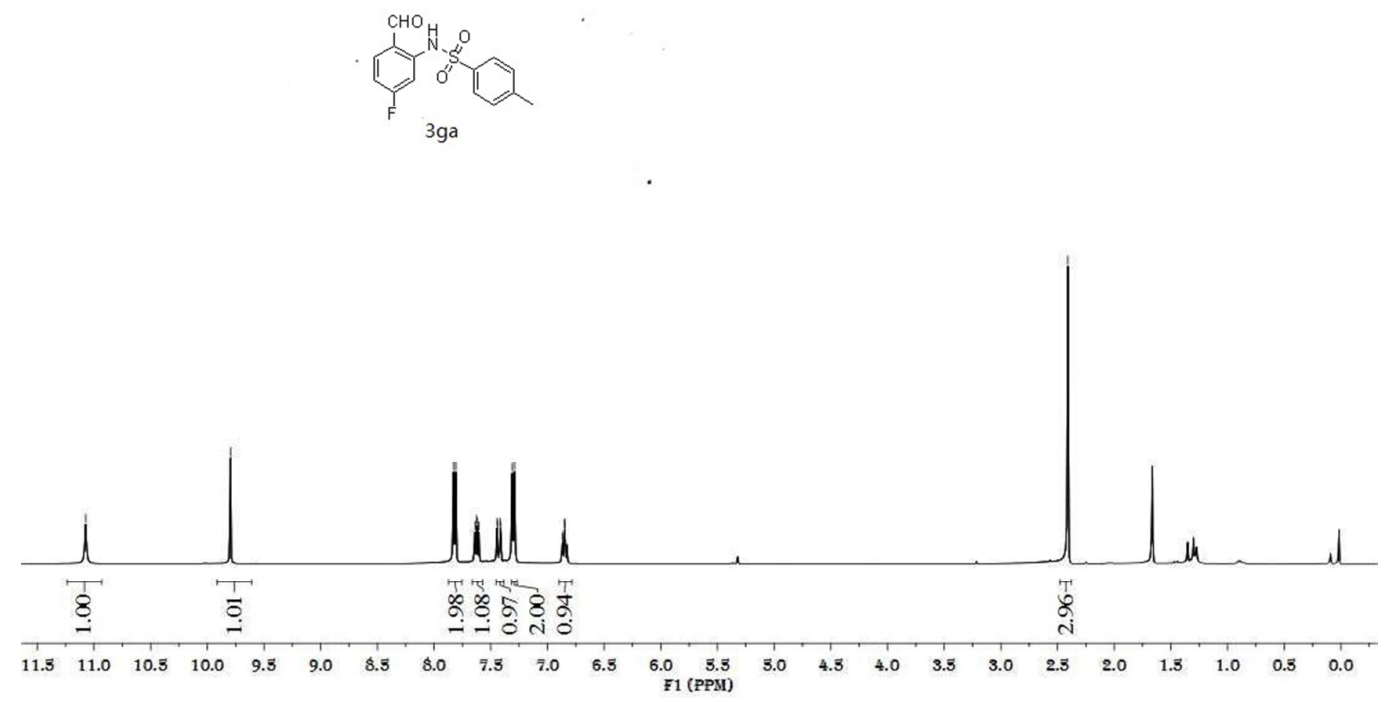

${ }^{13} \mathrm{C}$ NMR spectrum of $\mathbf{3 g a}$

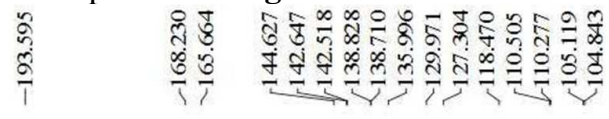

$\frac{\infty}{i}$

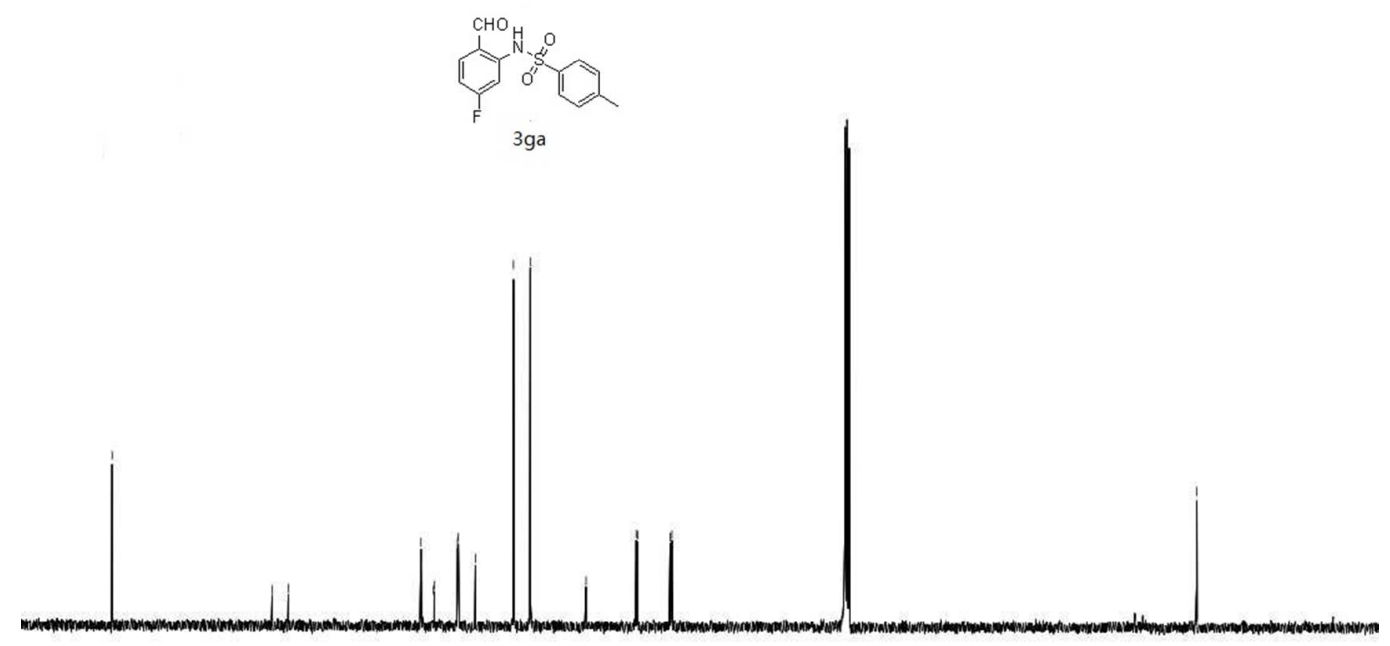

$\begin{array}{lllllllllllllllllllllllll} & 200 & 190 & 180 & 170 & 160 & 130 & 140 & 130 & 120 & 110 & 100 & 90 & 50 & 70 & 60 & 30 & 40 & 30 & 20 & 10 & 0\end{array}$ 
${ }^{1} \mathrm{H}$ NMR spectrum of 3 ha

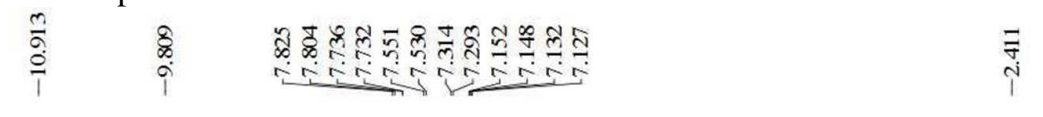
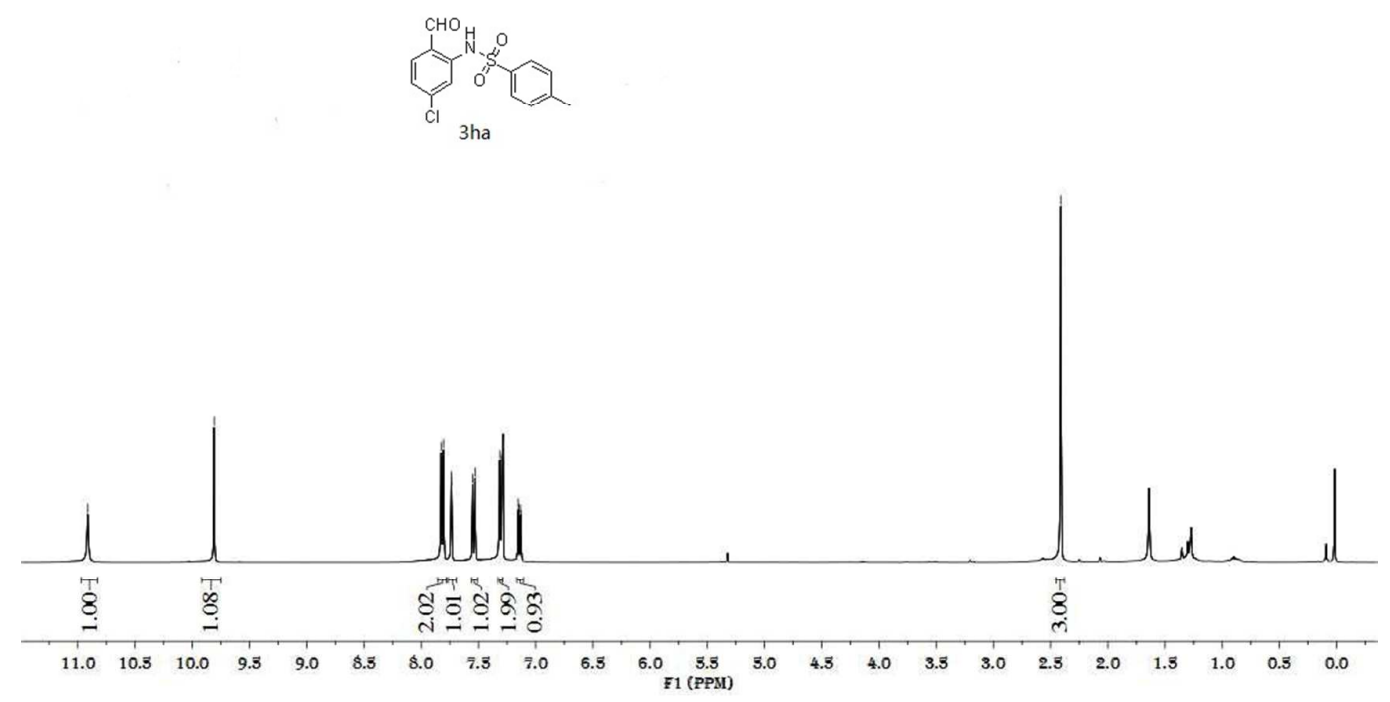

${ }^{13} \mathrm{C}$ NMR spectrum of $\mathbf{3 h a}$
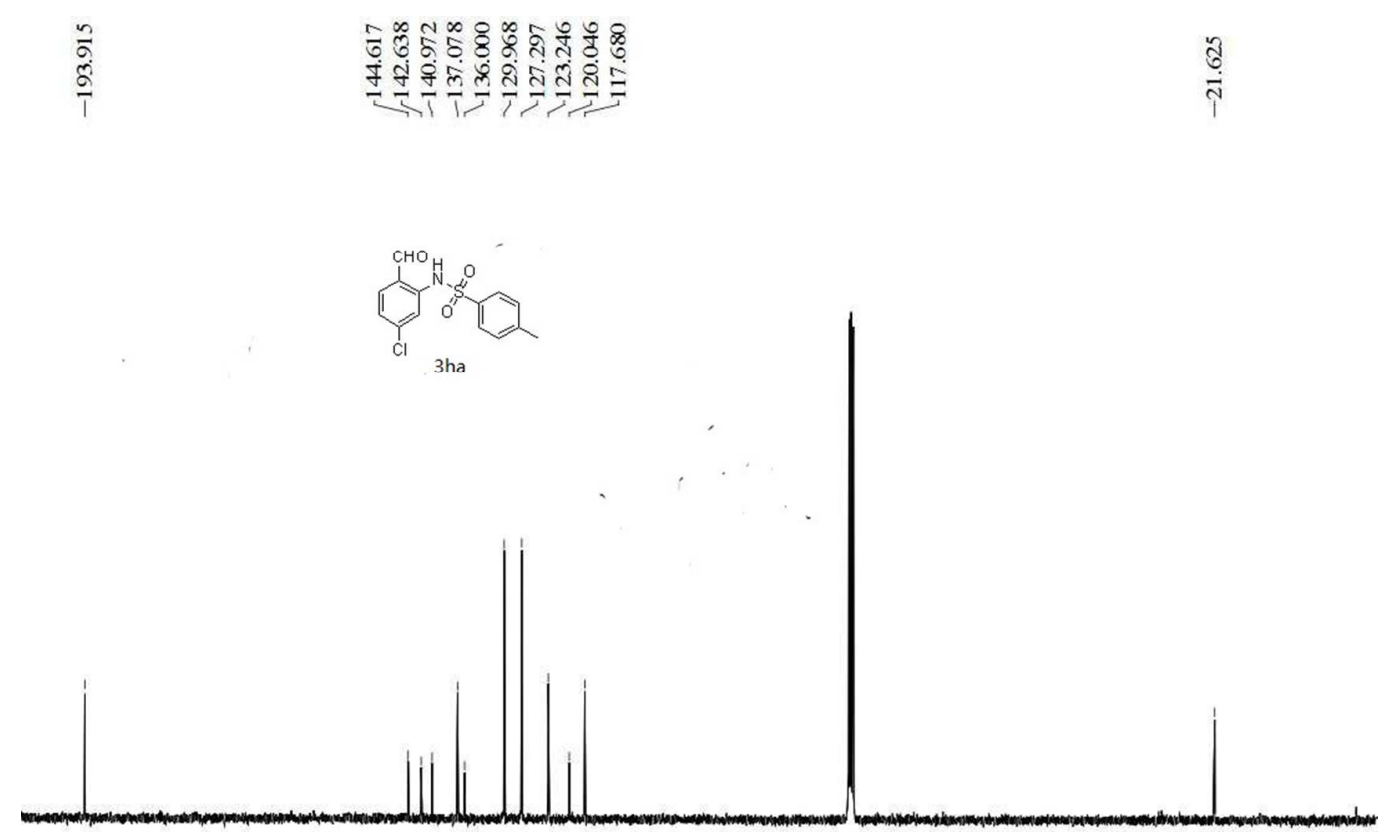

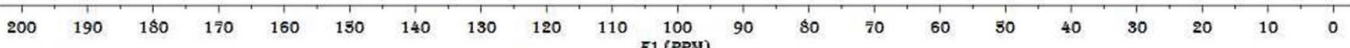


${ }^{1} \mathrm{H}$ NMR spectrum of 3ia

$\circ$
0
$i$

辛
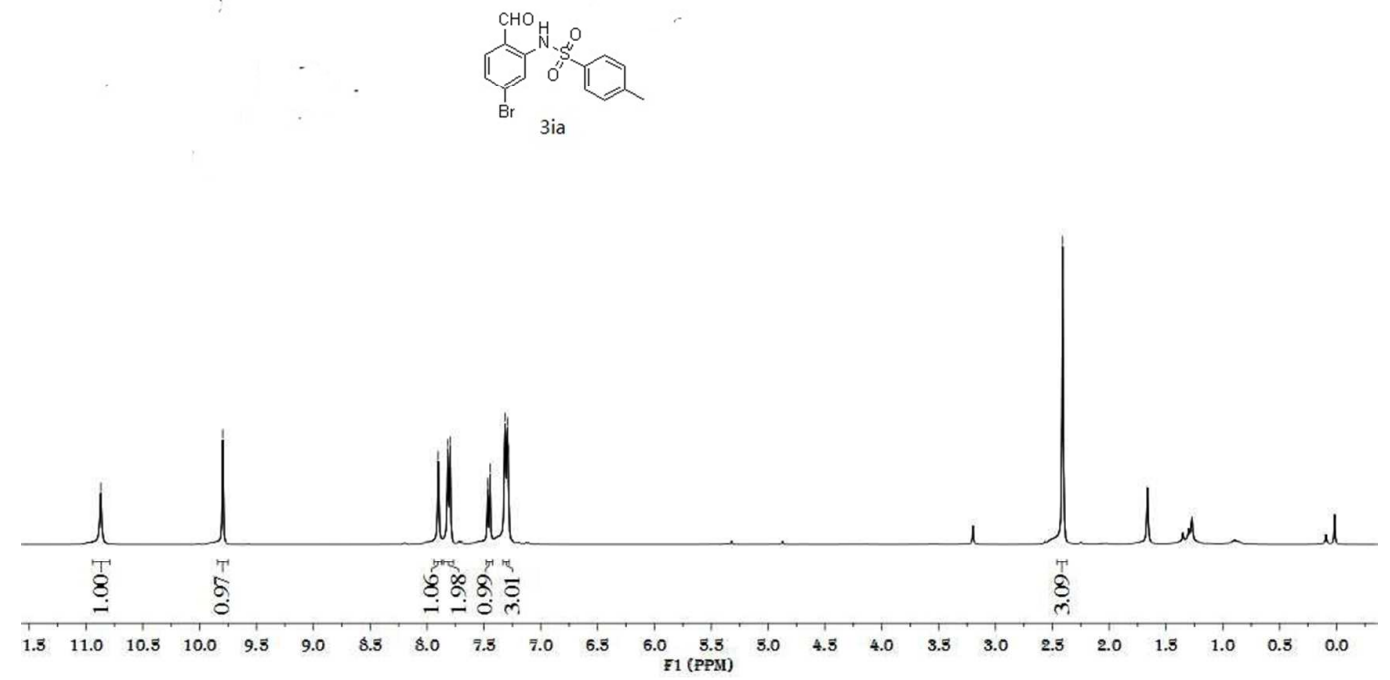

${ }^{13} \mathrm{C}$ NMR spectrum of 3 ia

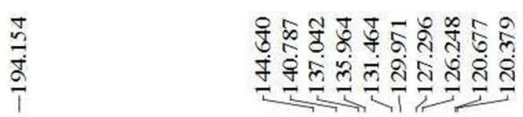

کु.

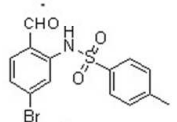

3ia

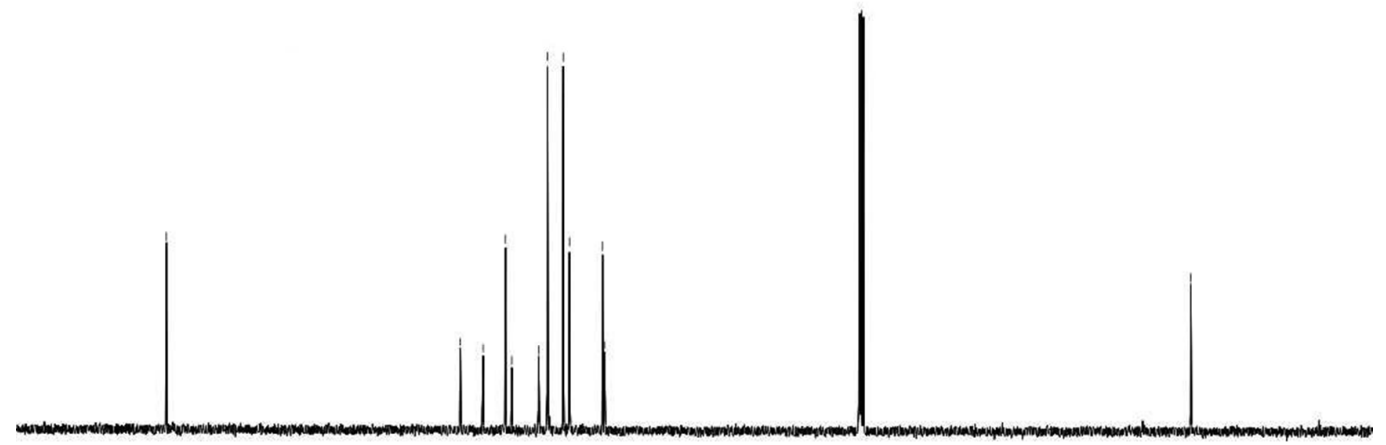

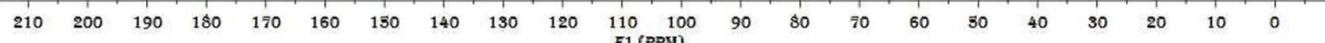


${ }^{1} \mathrm{H}$ NMR spectrum of $\mathbf{3 j a}$

$\begin{array}{lll}m & 0 \\ 0 & 0\end{array}$
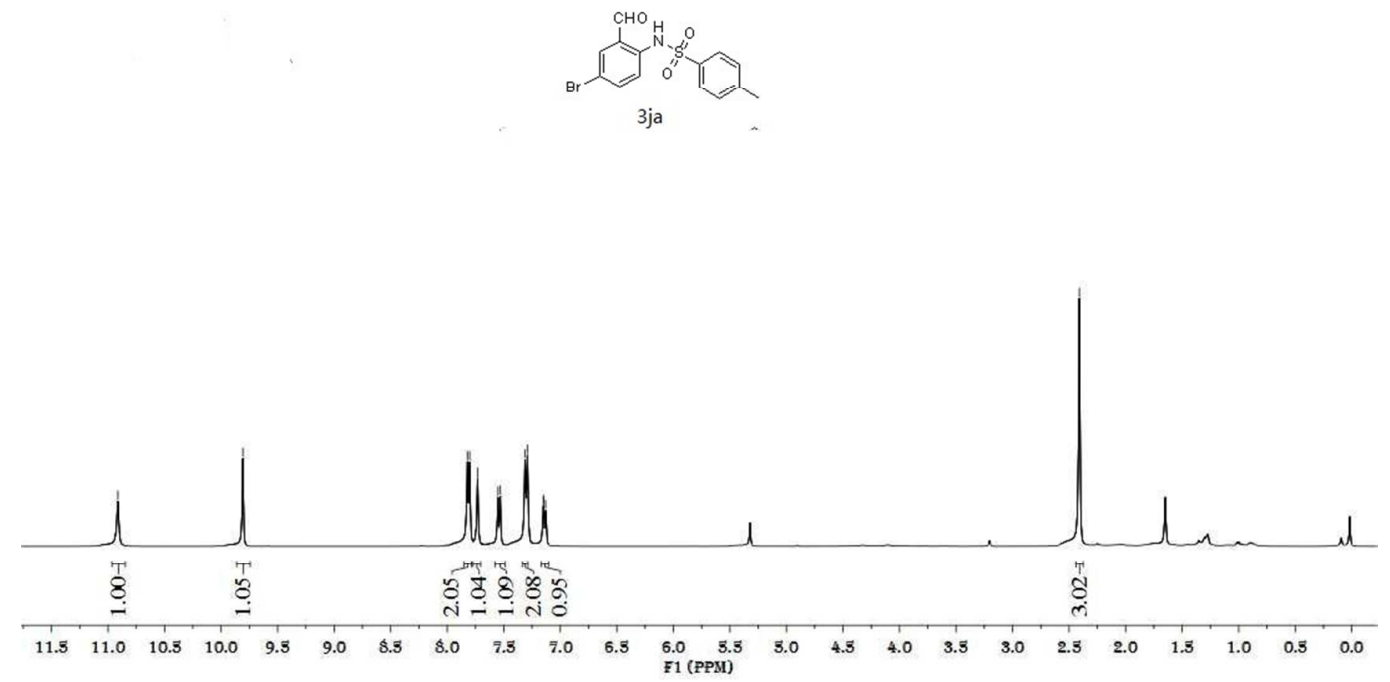

${ }^{13} \mathrm{C}$ NMR spectrum of $\mathbf{3 j a}$
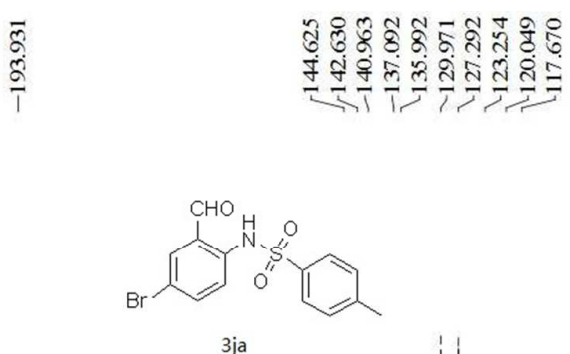

$3 \mathrm{ja}$

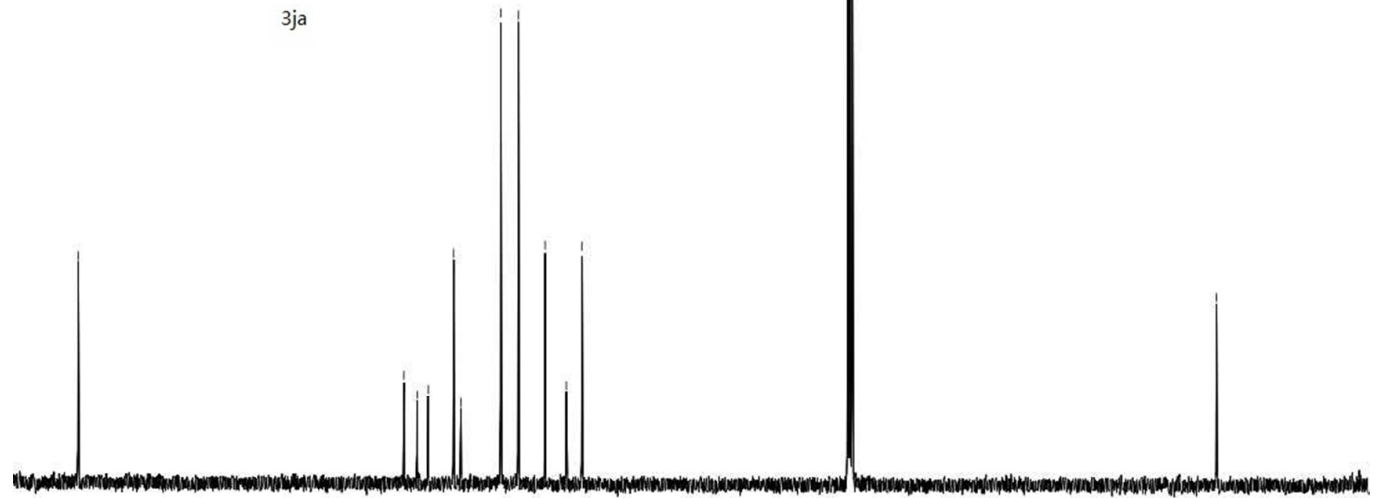

$200190 \quad 180 \quad 170 \quad 160 \quad 130 \quad 140 \quad 130 \quad 120 \quad 110 \quad \begin{array}{lllllllllllllllllllll}100 & 90 & 80 & 70 & 60 & 30 & 40 & 30 & 20 & 10 & 0\end{array}$ 
${ }^{1} \mathrm{H}$ NMR spectrum of $\mathbf{3 k a}$

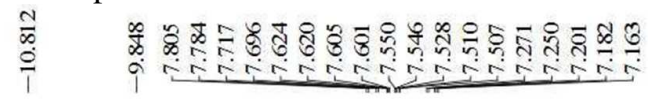
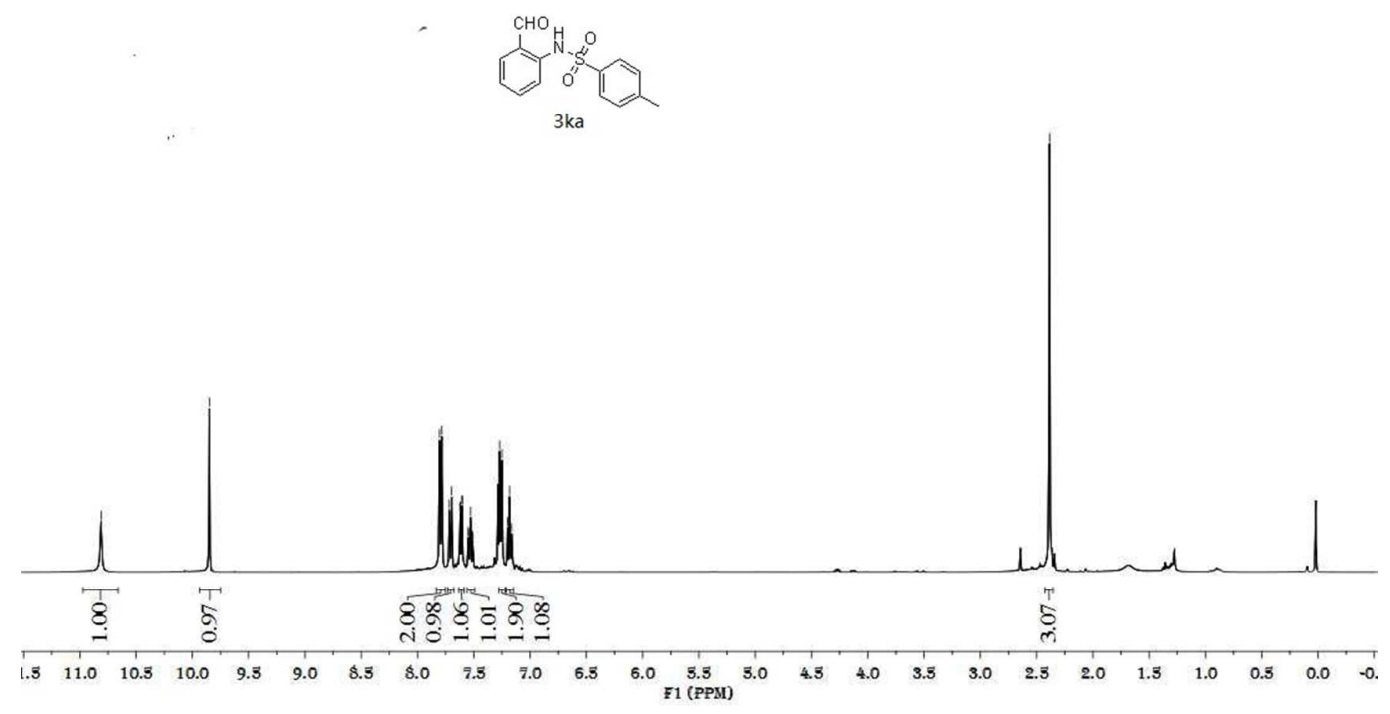

${ }^{13} \mathrm{C}$ NMR spectrum of $\mathbf{3 k a}$
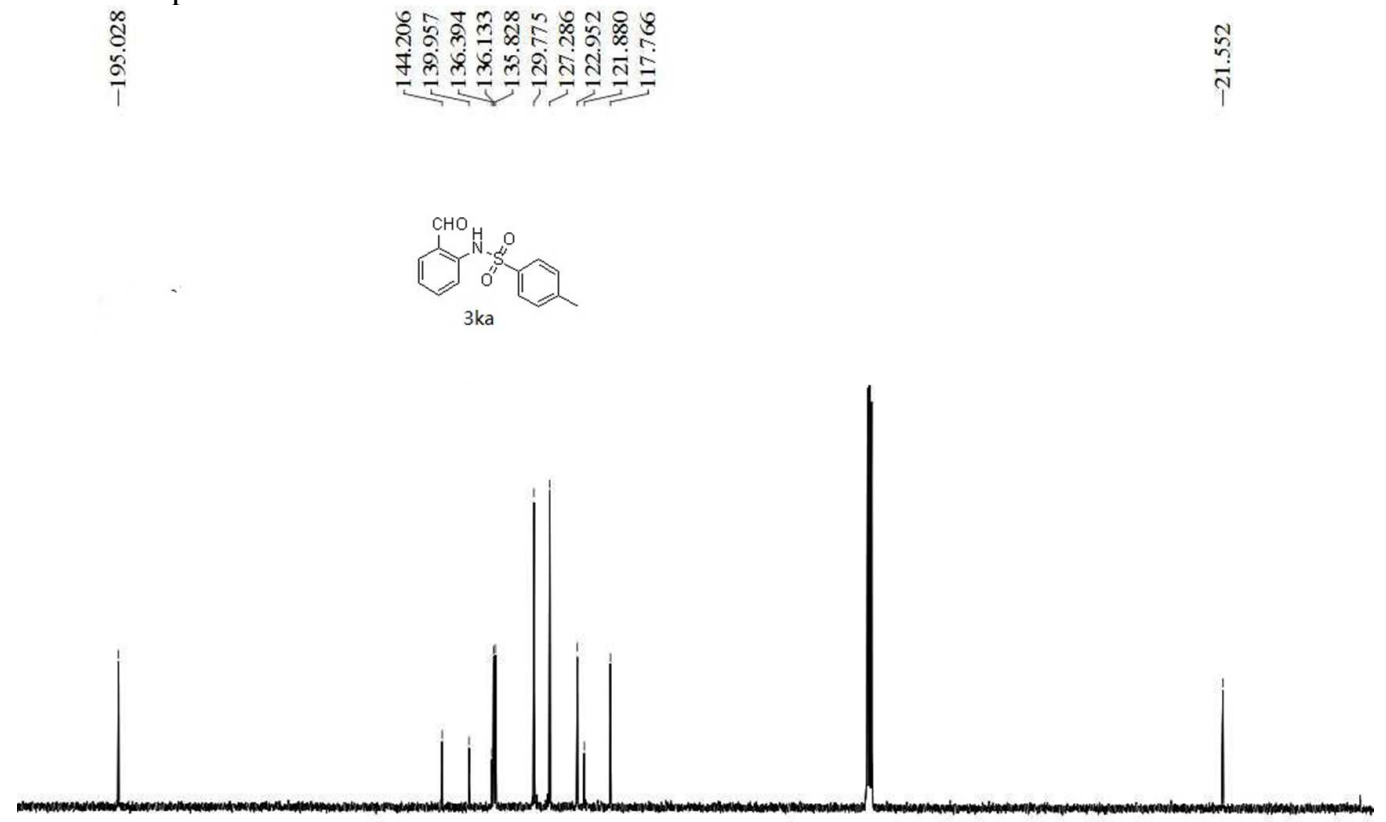

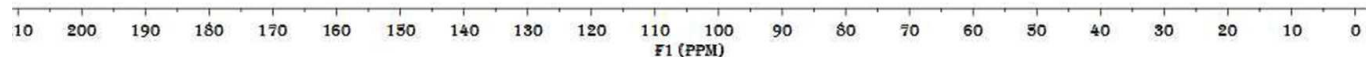


${ }^{1} \mathrm{H}$ NMR spectrum of $\mathbf{3 l a}$

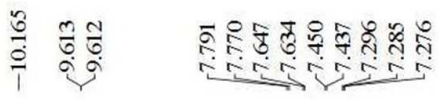
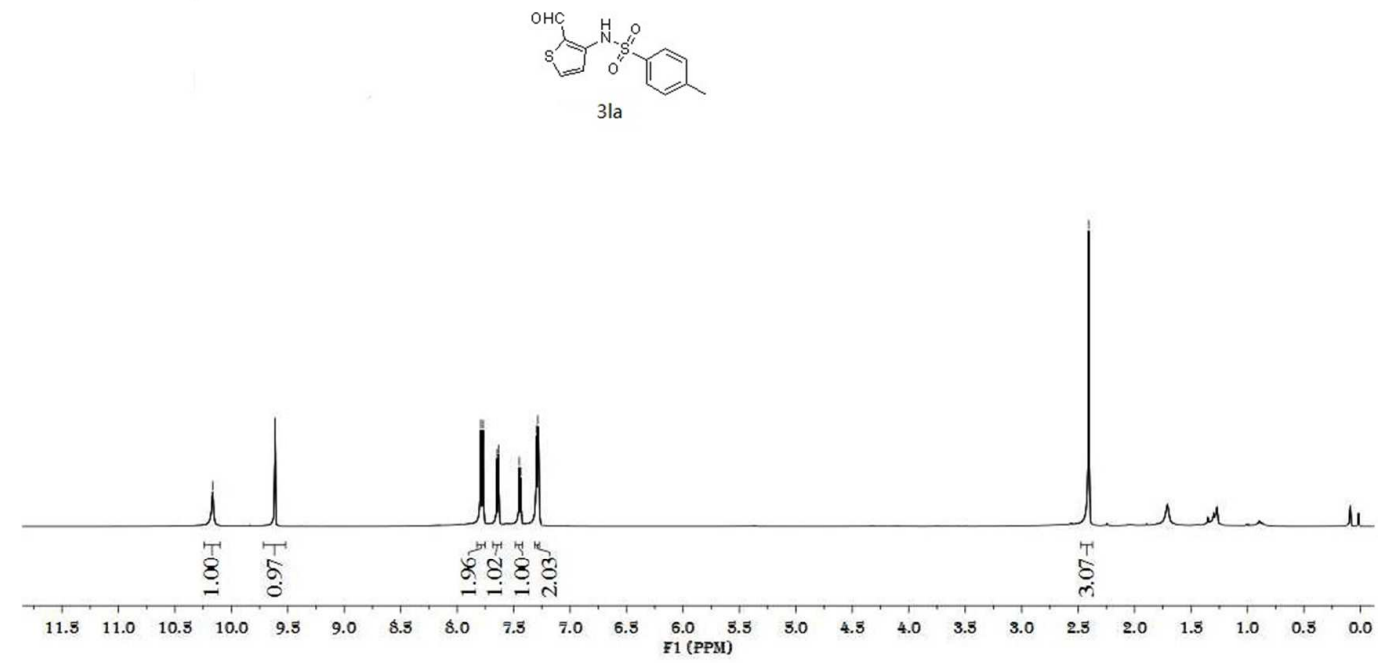

${ }^{13} \mathrm{C}$ NMR spectrum of $\mathbf{3 l a}$
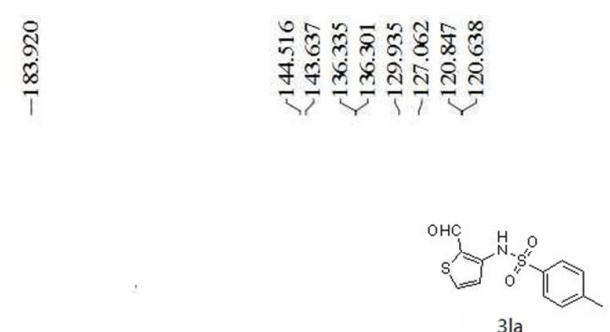

3la

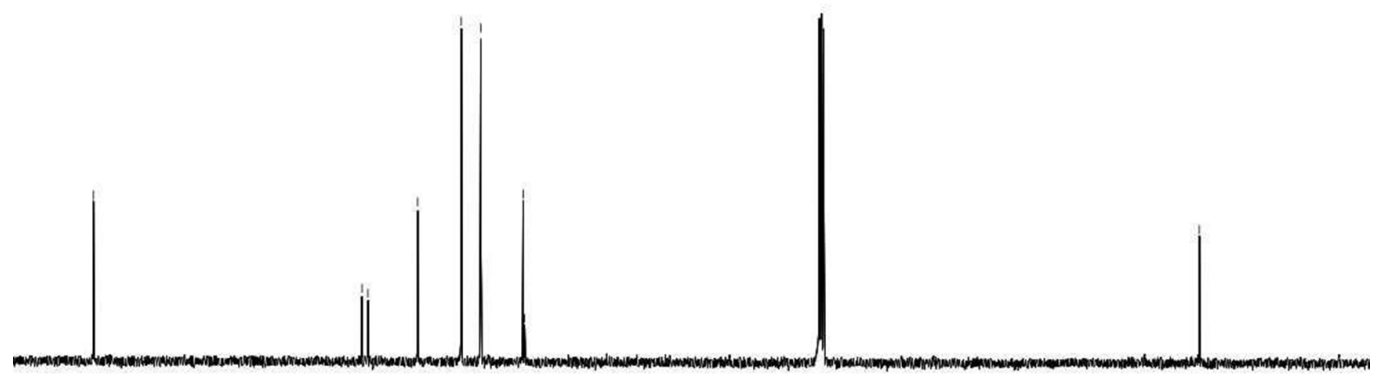

190

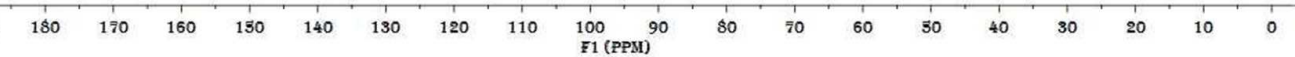


${ }^{1} \mathrm{H}$ NMR spectrum of $\mathbf{3 m a}$

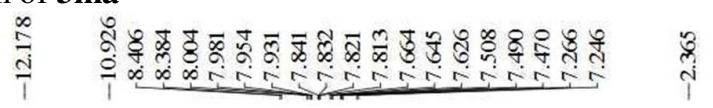
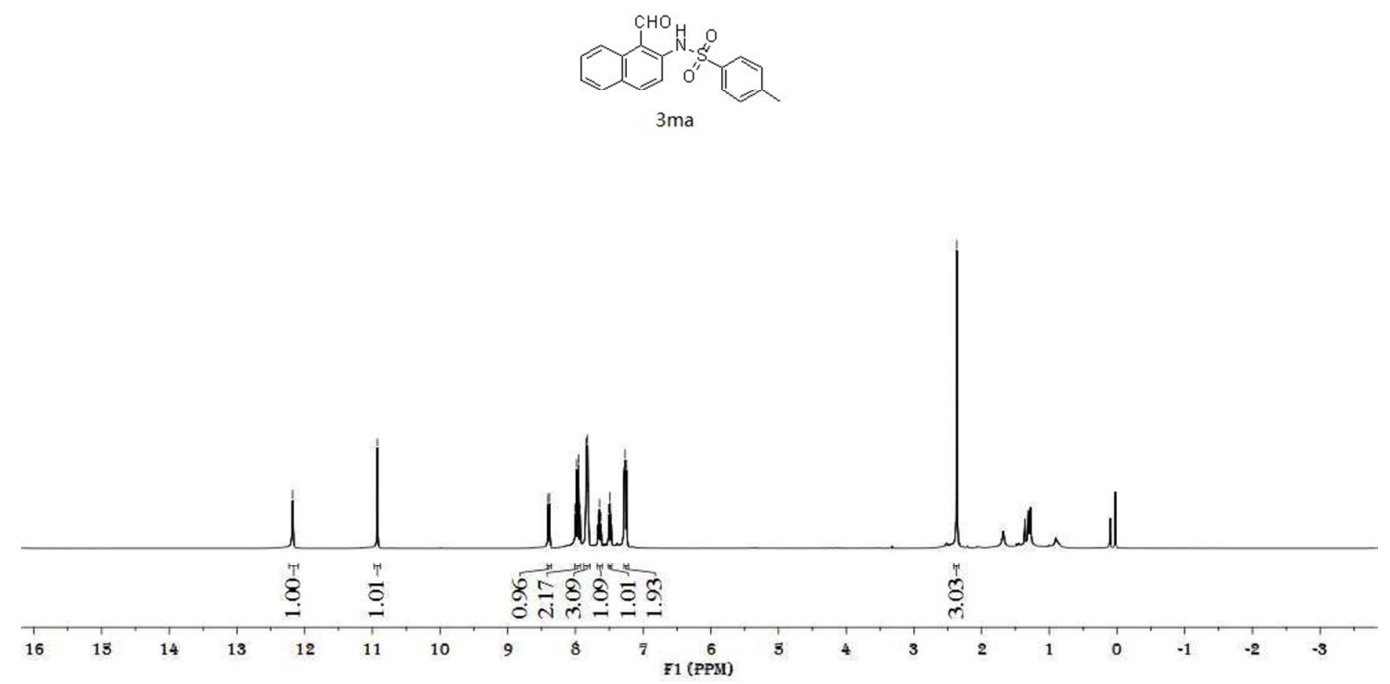

${ }^{13} \mathrm{C}$ NMR spectrum of $\mathbf{3 m a}$
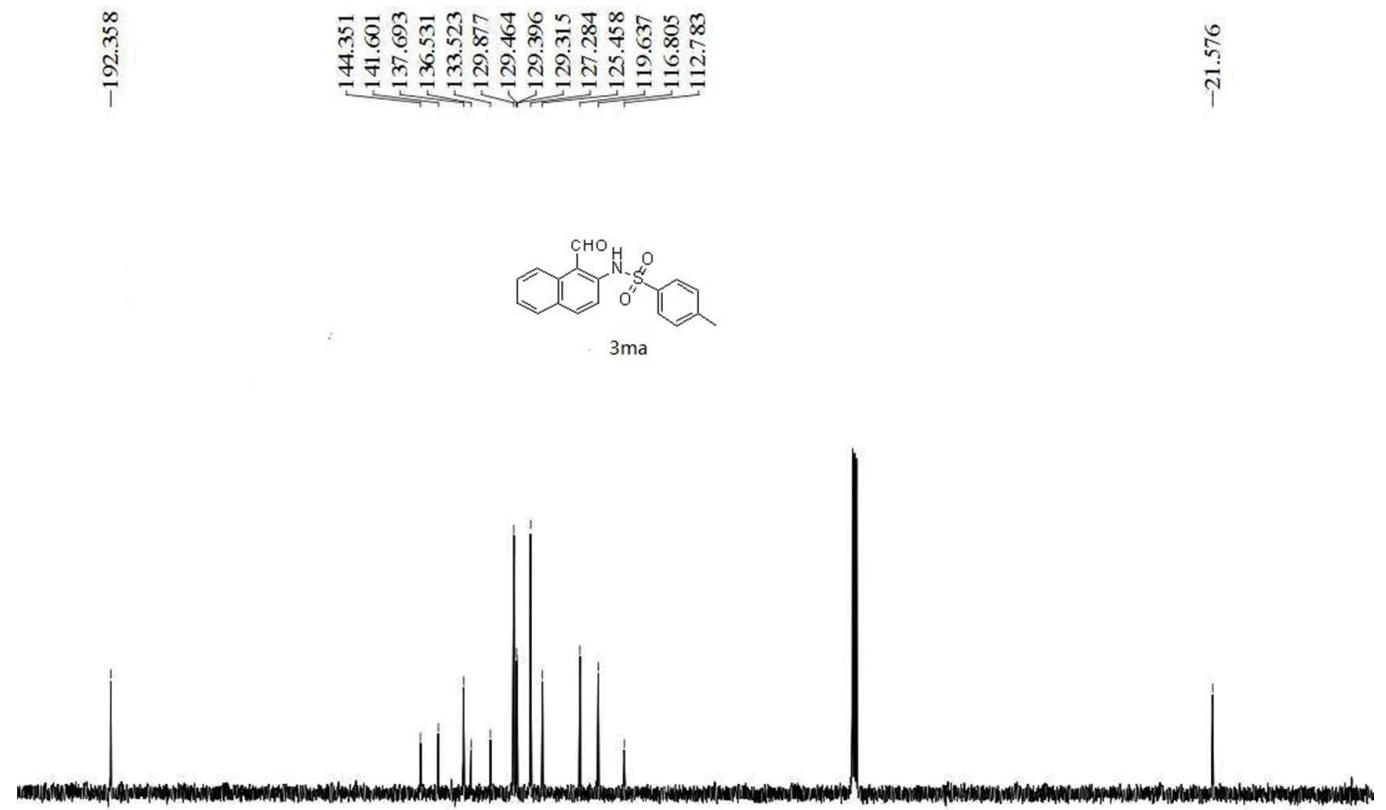

$\begin{array}{lllllllllll}200 & 190 & 180 & 170 & 160 & 150 & 140 & 130 & 120 & 110 & 100 \\ \text { F1 (PPM) }\end{array}$ 
The ${ }^{19} \mathrm{~F}$ NMR spectrum of 3ae

ปั
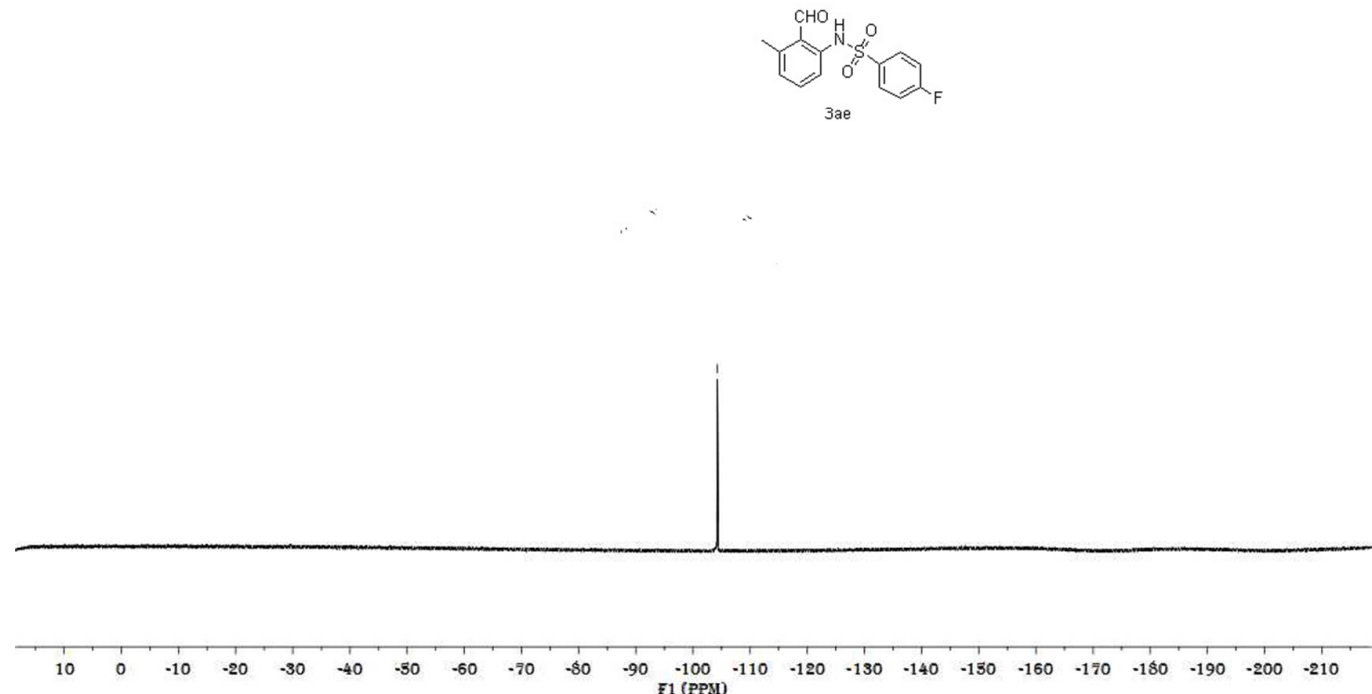

The ${ }^{19} \mathrm{~F}$ NMR spectrum of $\mathbf{3 b a}$

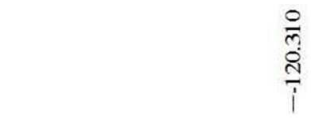

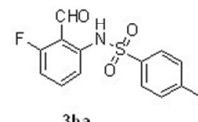

3 ba

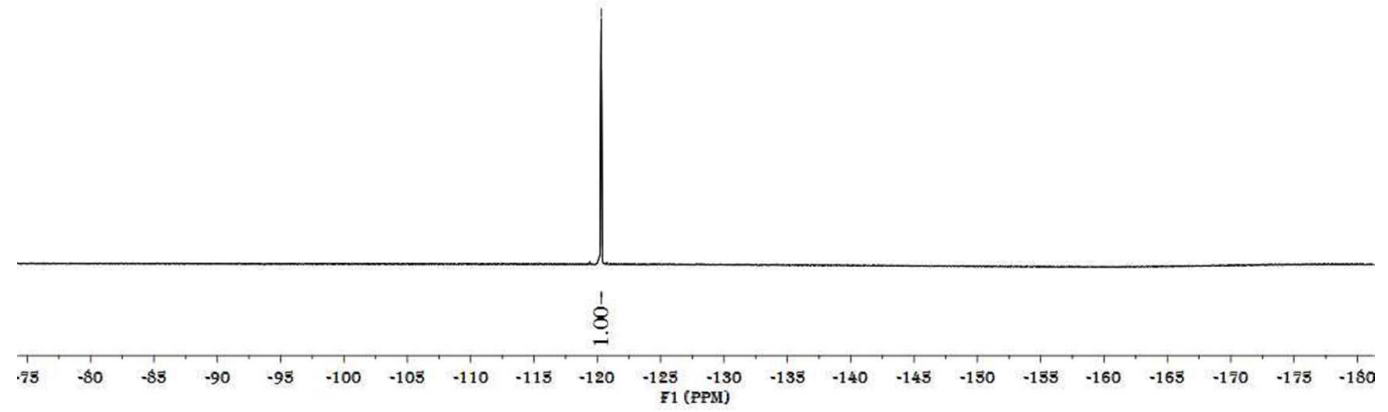


The ${ }^{19} \mathrm{~F}$ NMR spectrum of 3ea

$$
\text { ฺ̊ }
$$
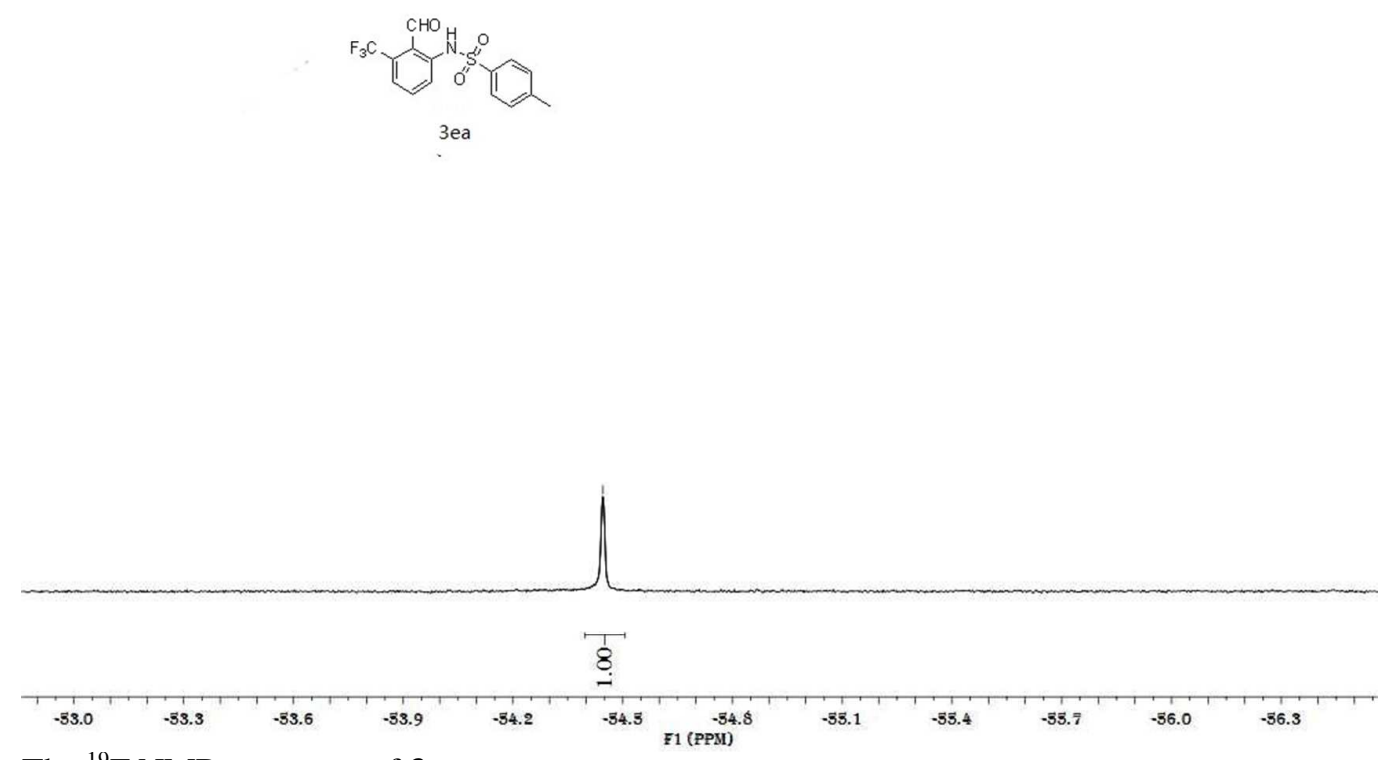

The ${ }^{19}$ F NMR spectrum of $\mathbf{3 g a}$
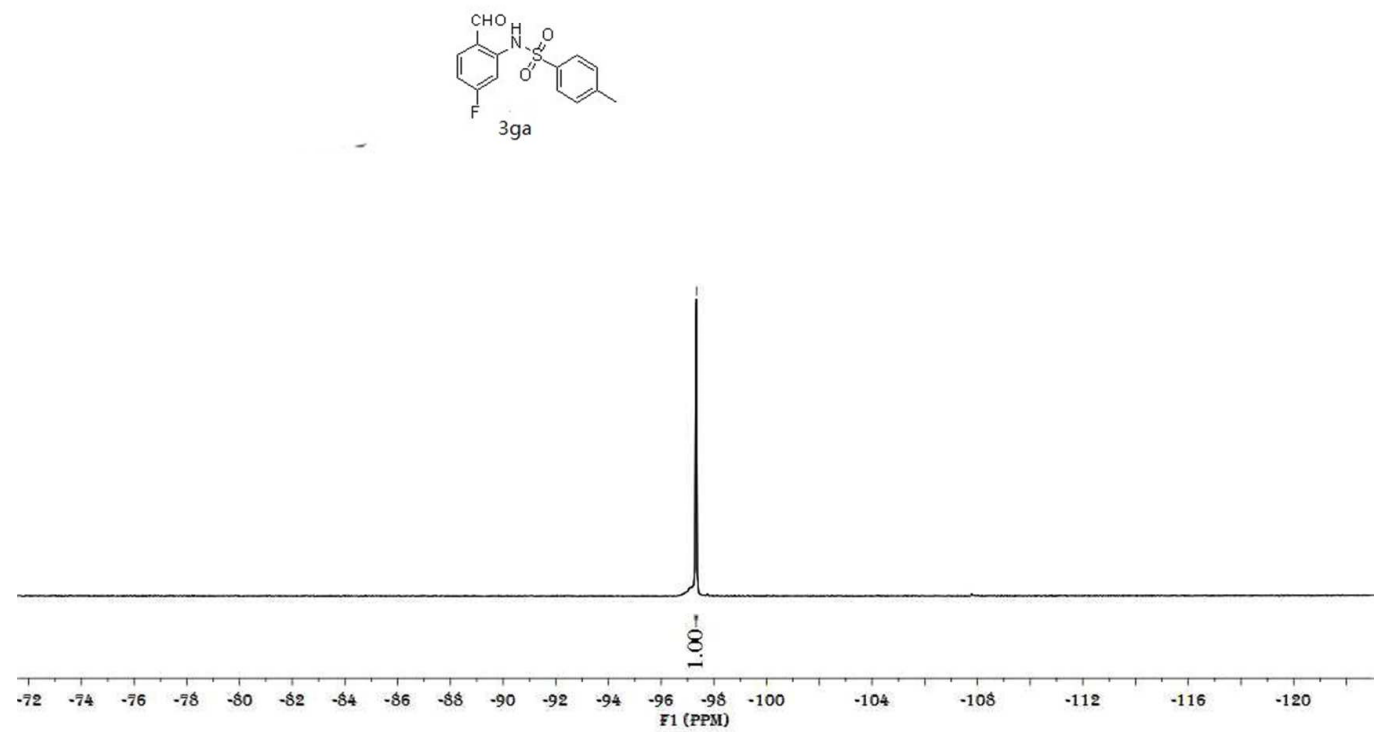